\title{
A Review of Error Estimation in Adaptive Quadrature
}

\author{
PEDRO GONNET \\ Dept. of Computer Science, ETH Zürich, Switzerland and \\ Mathematical Institute, University of Oxford, United Kingdom.
}

\begin{abstract}
The most critical component of any adaptive numerical quadrature routine is the estimation of the integration error. Since the publication of the first algorithms in the 1960s, many error estimation schemes have been presented, evaluated and discussed. This paper presents a review of existing error estimation techniques and discusses their differences and their common features. Some common shortcomings of these algorithms are discussed and a new general error estimation technique is presented.

Categories and Subject Descriptors: F.2.1 [Numerical Analysis]: Numerical Algorithms and Problems-Computations on polynomials; G.1.0 [Numerical Analysis]: General-Error analysis; Numerical algorithms; G.1.0 [Numerical Analysis]: Interpolation-Interpolation formulas; G.1.4 [Numerical Analysis]: Quadrature and Numerical Differentiation-Adaptive and iterative quadrature; Error analysis
\end{abstract}

General Terms: Algorithms, Reliability

Additional Key Words and Phrases: Numerical integration, Adaptive quadrature, Error estimation

\section{INTRODUCTION}

Adaptive quadrature, or adaptive numerical integration, refers to the process of approximating the integral of a given function to a specified precision by adaptively subdividing the integration interval into smaller sub-intervals over which a set of local quadrature rules are applied. Since the publication of the first adaptive quadrature routines almost 50 years ago [Morrin 1955; Villars 1956; Kuncir 1962], more than 20 distinct algorithms have been published, along several papers dedicated to their analysis [Casaletto et al. 1969; Hillstrom 1970; Kahaner 1971; Malcolm and Simpson 1975; Robinson 1979; Krommer and Überhuber 1998] and even on methodologies for their analysis [Lyness and Kaganove 1977].

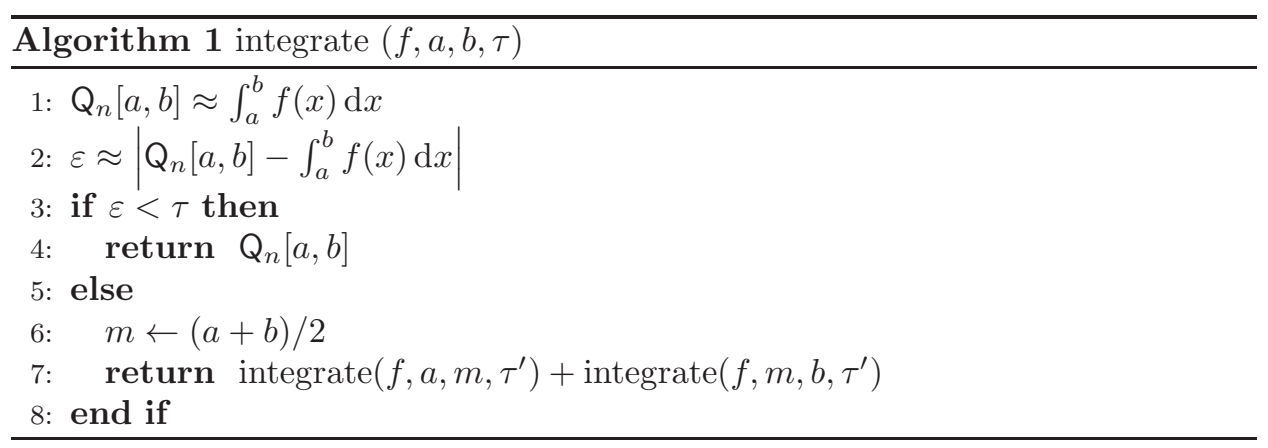

Many recursive adaptive quadrature routines follow the general scheme detailed ACM Computing Surveys, Vol. V, No. N, 20YY, Pages 1-0??. 
in Algorithm 1. In Line 1 an approximation $\mathrm{Q}_{n}[a, b]$ to the integral of $f(x)$ over $n$ points in the interval $[a, b]$ is computed and in Line 2 the error of this approximation is estimated. If this error is less than some user-specified local tolerance $\tau$ the algorithm returns the approximation $\mathrm{Q}_{n}[a, b]$. If the error is deemed too large, the interval is subdivided (in this example bisection is used) and the integration algorithm is applied recursively on both intervals separately for some new, adjusted tolerance $\tau^{\prime}$.

In the following, we will use $\mathrm{Q}_{n}[a, b]$ to denote a generic interpolatory quadrature rule over $n$ points in the interval $[a, b]$. For specific or well-known quadrature rules, we will use specific symbols such as $\mathrm{NC}_{n}[a, b]$ for Newton-Cotes, $\mathrm{CC}_{n}[a, b]$ for Clenshaw-Curtis and $\mathrm{G}_{n}[a, b]$ and $\mathrm{GK}_{n}[a, b]$ for Gauss and Gauss-Kronrod rules over $n$ points respectively. We will use the notation $\mathrm{Q}_{n}^{(m)}[a, b]$ to denote the quadrature rule $Q_{n}$ applied on $m$ panels of equal size in $[a, b]$. In [Davis and Rabinowitz 1967] $\mathrm{Q}_{n}^{(m)}[a, b]$ is referred to as a compound or composite quadrature rule. We will call $m$ the multiplicity of $\mathbf{Q}_{n}^{(m)}[a, b]$.

A slightly different approach to Algorithm 1, motivated by the desire for a sharper global error estimate and better interval selection criteria - and partially due to the unavailability of recursion in early computer programming languages - is shown in Algorithm 2. In this non-recursive approach, a heap of intervals, sorted by their local error estimates, is maintained (Line 3). As long as the sum of the individual error estimates is larger than the required global tolerance $\tau$ (Line 4), the interval at the top of the heap (i.e. the interval with the largest error estimate, Line 5) is subdivided (Line 6). The resulting subintervals are evaluated (Lines 7 to 10) and returned to the heap (Lines 13 and 14), and the global integral and global error estimate are updated (Lines 11 and 12).

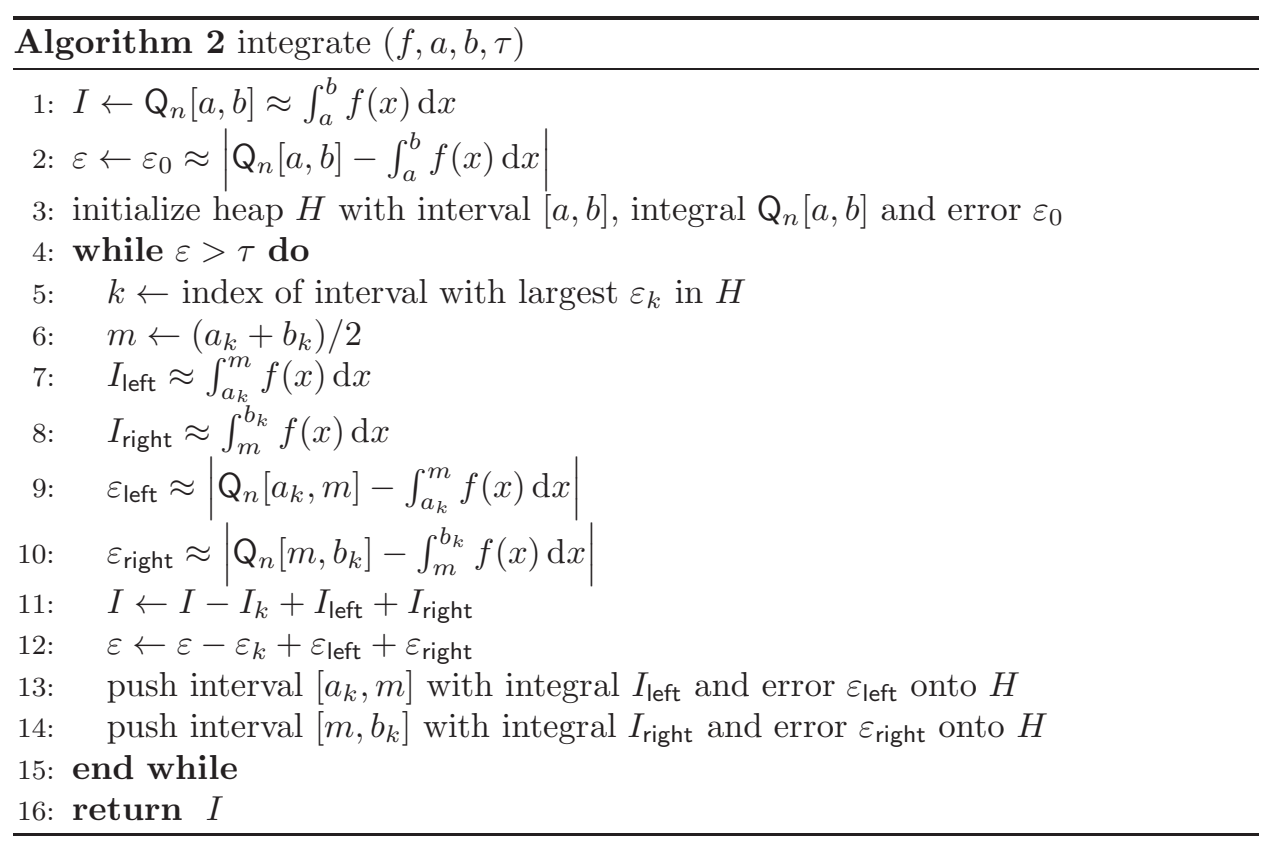


If the integrand is Riemann integrable and the error estimates are exact, both Algorithm 1 and Algorithm 2 will converge to the exact integral. It is therefore only failures in the estimation of the integration error that will cause the quadrature algorithms to fail. It is for this reason that inn this review, we will concentrate only on the error estimate

$$
\varepsilon \approx\left|Q_{n}[a, b]-\int_{a}^{b} f(x) \mathrm{d} x\right| .
$$

as it is computed in Line 2 of Algorithm 1 and Lines 2, 9 and 10 of Algorithm 2.

We will distinguish between the local and global error of an adaptive quadrature routine. During adaptive integration, the interval is subdivided into sub-intervals $\left[a_{k}, b_{k}\right]$ with $a \leq a_{k}<b_{k} \leq b$. This subdivision occurs either recursively (as in Line 7 of Algorithm 1) or explicitly (as in Lines 13-14 of Algorithm 2). The local error $\varepsilon_{k}$ of the $k^{\text {th }}$ interval $\left[a_{k}, b_{k}\right]$ and the global error $\varepsilon$ are defined as

$$
\varepsilon_{k}=\left|\mathrm{Q}_{n}\left[a_{k}, b_{k}\right]-\int_{a_{k}}^{b_{k}} f(x) \mathrm{d} x\right| \quad \text { and } \varepsilon=\left|\sum_{k} \mathrm{Q}_{n}\left[a_{k}, b_{k}\right]-\int_{a}^{b} f(x) \mathrm{d} x\right| .
$$

The sum of the local errors forms an upper bound for the global error $\left(\varepsilon \leq \sum_{k} \varepsilon_{k}\right)$.

We further distinguish between the absolute errors (1), the locally relative error and the globally relative local error

$$
\varepsilon_{k}^{(\text {Irel })}=\left|\frac{\mathrm{Q}_{n}\left[a_{k}, b_{k}\right]-\int_{a_{k}}^{b_{k}} f(x) \mathrm{d} x}{\int_{a_{k}}^{b_{k}} f(x) \mathrm{d} x}\right|, \quad \varepsilon_{k}^{(\text {grel })}=\left|\frac{\mathrm{Q}_{n}\left[a_{k}, b_{k}\right]-\int_{a_{k}}^{b_{k}} f(x) \mathrm{d} x}{\int_{a}^{b} f(x) \mathrm{d} x}\right| .
$$

We also define the global relative error which is bounded by the sum of the globally relative local errors:

$$
\varepsilon=\frac{\left|\sum_{k} Q_{n}\left[a_{k}, b_{k}\right]-\int_{a}^{b} f(x) \mathrm{d} x\right|}{\int_{a}^{b} f(x) \mathrm{d} x} \leq \sum_{k}\left|\frac{Q_{n}\left[a_{k}, b_{k}\right]-\int_{a_{k}}^{b_{k}} f(x) \mathrm{d} x}{\int_{a}^{b} f(x) \mathrm{d} x}\right| .
$$

The sum of the locally relative errors, however, form no such bound.

In the following, we will often refer to the degree of a quadrature rule. A quadrature rule is of degree $n$ when it integrates all polynomials of degree $\leq n$ exactly, but not all polynomials of degree $n+1$. This is synonymous with the precise degree of exactness as defined by Gautschi [2004] or the degree of accuracy as defined by Krommer and Überhuber [1998]. If a quadrature rule is of degree $n$, then its order of accuracy as defined by Skeel and Keiper [1993], to which we will simply refer to as its order, is $n+1$.

The goal of this review is to analyze and compare different error estimation techniques qualitatively, similarly to the analysis by Laurie [1985]. We will start with an overview of the most significant contributions over the last 50 years. Following this analysis, we will present a new error estimator which overcomes most of the problems observed in previous error estimators.

In the following two sections we will discuss existing linear (Section 2) and nonlinear (Section 3) error estimation techniques ${ }^{1}$. In Section 4 a new error estimation

\footnotetext{
${ }^{1}$ For a more detailed review, see [Gonnet 2009a].
} 
technique is presented and its relation to previous error estimators is discussed. In Section 5 we will apply the discussed error estimators to a number of test functions to assess their performance. In Section 6 we discuss these results and try to interpret them qualitatively.

\section{LINEAR ERROR ESTIMATORS}

In this section we will look at a number of linear error estimators. We define a linear error estimator as an estimate computed from a linear combination of evaluations of the integrand. Such estimators can be quadrature-like rules, linear combinations or differences of quadrature rules or quantities computed using linear extrapolation techniques, e.g. the Romberg scheme.

\subsection{Early Error Estimators Based on Rules of Equal Degree}

There seems to be some confusion as to who actually published the first adaptive quadrature algorithm. Davis and Rabinowitz [1967] cite the works of Villars [1956], Henriksson [1961] and Kuncir (see Section 2.1).

Although no explicit attribution is given, Henriksson's algorithm seems to be an unmodified ALGOL-implementation of the algorithm described by Villars which is, as the author himself states, only a slight modification of a routine developed by Morrin [1955, cited in Villars 1956] in 1955. These three algorithms are more reminiscent of ODE-solvers, integrating the function stepwise from left to right using Simpson's rule and adapting (doubling or halving) the step-size whenever an estimate converges or fails to do so. In doing so they effectively discard function evaluations and so lose information on the structure of the integrand. We will therefore not consider them to be "genuine" adaptive integrators.

In 1962, Kuncir [1962] publishes the first adaptive quadrature routine ${ }^{2}$ following the scheme in Algorithm 1 and using the locally relative local error estimate

$$
\varepsilon_{k}=\left|\frac{\mathrm{S}^{(1)}\left[a_{k}, b_{k}\right]-\mathrm{S}^{(2)}\left[a_{k}, b_{k}\right]}{\mathrm{S}^{(2)}\left[a_{k}, b_{k}\right]}\right|
$$

where $\mathbf{S}^{(1)}\left[a_{k}, b_{k}\right]$ is Simpson's rule applied over the entire interval $\left[a_{k}, b_{k}\right]$ and $\mathrm{S}^{(2)}\left[a_{k}, b_{k}\right]$ is Simpson's rule applied on the sub-intervals $\left[a, \frac{a+b}{2}\right]$ and $\left[\frac{a+b}{2}, b\right]$. If the error estimate is below the required tolerance, the estimate $\mathrm{S}^{(2)}\left[a_{k}, b_{k}\right]$ is used as the local approximation to the integral.

The error estimate is based on the assumption that if the estimate $\mathrm{S}^{(2)}\left[a_{k}, b_{k}\right]$ is a better approximation of the integral than $\mathrm{S}^{(1)}\left[a_{k}, b_{k}\right]$, the difference between both estimates will be a good estimate of the difference between $\mathbf{S}^{(1)}\left[a_{k}, b_{k}\right]$ and the actual integral.

Replacing every evaluation of the integrand in the un-scaled error estimate (3) with an appropriate $f(a+h)$ and expanding it in a Taylor expansion around $a$, as

\footnotetext{
${ }^{2}$ Although Kuncir predates McKeeman by about half a year, many publications [Espelid 2007; 2002; 2004a; 2003; Berntsen and Espelid 1991; Malcolm and Simpson 1975], credit McKeeman with having published the first adaptive integrator. Interestingly enough, the very similar works of both Kuncir and McKeeman were both published in the same journal (Communications of the ACM) in the same year (1962) in different issues of the same volume (Volume 5), both edited by the same editor (J.H. Wegstein). This duplication of efforts does not seem to have been noticed at the time.

ACM Computing Surveys, Vol. V, No. N, 20YY,
} 
is done in [Gander 2006], we obtain

$$
\mathrm{S}^{(1)}\left[a_{k}, b_{k}\right]-\mathrm{S}^{(2)}\left[a_{k}, b_{k}\right]=\frac{\left(b_{k}-a_{k}\right)^{5}}{3072} f^{(4)}(\xi), \quad \xi \in\left[a_{k}, b_{k}\right] .
$$

Inserting the Taylor expansion into the actual error gives a similar result:

$$
\mathrm{S}^{(2)}\left[a_{k}, b_{k}\right]-\int_{a_{k}}^{b_{k}} f(x) \mathrm{d} x=\frac{\left(b_{k}-a_{k}\right)^{5}}{46080} f^{(4)}(\xi), \quad \xi \in\left[a_{k}, b_{k}\right] .
$$

If we assume that $f^{(4)}(x)$ is more or less constant for $x \in\left[a_{k}, b_{k}\right]$ and both (4) and (5) therefore have similar values for $f^{(4)}(\xi)$, then the error estimate is actually 15 times larger than the actual integration error. This factor of 15 might seem large, but in practice it is a good guard against bad estimates when $f^{(4)}(x)$ is not constant for $x \in\left[a_{k}, b_{k}\right]$.

In the same year, McKeeman [1962] publishes a similar recursive algorithm (following Algorithm 1, yet using trisection instead of bisection) using the globally relative local error estimate

$$
\varepsilon_{k}=\frac{1}{\hat{I}}\left|\mathrm{~S}^{(1)}\left[a_{k}, b_{k}\right]-\mathrm{S}^{(3)}\left[a_{k}, b_{k}\right]\right|
$$

where $\hat{I}$ is an approximation to the global integral of the absolute value of $f(x)$.

Using the same analysis as in (4), we can compute the ratio of the computed and exact errors and obtain

$$
\left|\frac{\mathrm{S}^{(1)}[a, b]-\mathrm{S}^{(3)}[a, b]}{\mathrm{S}^{(3)}[a, b]-\int_{a}^{b} f(x) \mathrm{d} x}\right| \approx 80
$$

i.e. the error is overestimated by a factor of 80 for sufficiently smooth ${ }^{3}$ integrand.

The use of a globally relative local error estimate is an important improvement. Besides forming a correct upper bound for the global error, it does not run into problems in sub-intervals where the integrand approaches 0, causing any locally relative error estimate to approach infinity. The use of an error relative to the global integral of the absolute value of the function is a good guard against cancellation or smearing [Henrici 1982] when summing-up the integrals over the sub-intervals.

A year later, McKeeman and Tesler [1963] publish a non-recursive ${ }^{4}$ version of of the integrator with a better local tolerance computation and shortly thereafter, McKeeman publishes another recursive adaptive integrator [McKeeman 1963] based on Newton-Cotes rules over a set of $n$ points, where $n$ is a user-defined parameter. In the same vein as the previous integrator, the following error estimate is used

$$
\varepsilon_{k}=\frac{1}{\hat{I}_{d}}\left|\mathrm{NC}_{n}^{(1)}\left[a_{k}, b_{k}\right]-\mathrm{NC}_{n}^{(n-1)}\left[a_{k}, b_{k}\right]\right|
$$

\footnotetext{
${ }^{3}$ In the following, we will use the rather loose expression "sufficiently smooth" when, for a quadrature rule of order $n$, the $n$th derivative of the integrand is sufficiently close to constant in the integration interval, such that the error estimate will not fail.

${ }^{4}$ Their algorithm is non-recursive in the sense that an explicit stack is maintained, analogous to the one generated in memory during recursion, and not as in the scheme presented in Algorithm 2
} 
At every recursion level, the interval is subdivided into $n-1$ panels and, if the tolerance is met, the value of $\mathrm{NC}_{n}^{(n-1)}[a, b]$ is used as an approximation to the integral.

Replacing the evaluations of the integrand $f(a+h)$ by their Taylor expansions around $a$ and inserting them into the ratio of the computed and exact error as in (7), we can see that for $n=3$ (i.e. applying Simpson's rule), we overestimate the actual error by a factor of 15 . For $n=4$, this factor grows to 80 , as observed for McKeeman's first integrator (see (7)). For $n=5$ it is 4095 and for $n=8$, the maximum allowed in the algorithm, it is 5764800 (7 decimal digits!), making this a somewhat strict estimate both in theory and in practice.

In 1969, Lyness [1969] publishes the first rigorous analysis of McKeeman's integrator and implements a revised algorithm, SQUANK[Lyness 1970]. He suggests using the absolute local error instead of the globally relative local error, bisection instead of trisection and includes the resulting factor of 15 in the error estimate ${ }^{5}$ :

$$
\varepsilon_{k}=\frac{1}{15}\left|\mathrm{~S}^{(1)}\left[a_{k}, b_{k}\right]-\mathrm{S}^{(2)}\left[a_{k}, b_{k}\right]\right|
$$

He further suggests using Romberg extrapolation to compute the five-node NewtonCotes formula from the two Simpson's approximations ${ }^{6}$ :

$$
\mathrm{NC}_{5}^{(1)}[a, b]=\frac{1}{15}\left(16 \mathrm{~S}^{(2)}[a, b]-\mathrm{S}^{(1)}[a, b]\right) .
$$

This is a departure from previous methods, in which the error estimate and the integral approximation were of the same degree, making it impracticable to relate the error estimate to the integral approximation without making additional assumptions on the smoothness of the integrand.

In a 1975 paper, Malcolm and Simpson [1975] present a global version of SQUANK called SQUAGE (Simpson's Quadrature Used Adaptively Global Error) along the lines of Algorithm 2, and conclude that global adaptivity allows for better control of the error estimate ${ }^{7}$.

In 1977, Forsythe et al. [1977] publish the recursive quadrature routine QUANC8, which uses essentially the same basic error estimate as Lyness (9), yet using NewtonCotes rules over 9 points, resulting in a scaling factor of 1023 instead of 15 (see (9)). Analogously to (10), the two quadrature rules are combined using Romberg extrapolation to compute a 11th degree approximation which is used as the approximation to the integral ${ }^{8}$.

The same approach, although effectively evaluated differently, was later re-used

\footnotetext{
${ }^{5}$ Note that McKeeman's original error estimate was off by a factor of 80 (see (7)). The factor of 15 comes from using bisection instead of trisection.

${ }^{6}$ Interestingly enough, this was already suggested by Villars [1956] and implemented by Henriksson [1961], but apparently subsequently forgotten.

${ }^{7}$ In their paper, Malcolm and Simpson state (erroneously) that Lyness' SQUANK uses $S^{(2)}[a, b]$ as its approximation to the integral and, as their results suggest, $S^{(2)}[a, b]$ was also used in their implementation thereof. This omission, however, has no influence on their results or the conclusions they draw in their paper as they only consider the number of intervals generated by the global and local error estimates, and not the accuracy of the final result.

${ }^{8}$ This routine was integrated into MATLAB as quad8, albeit without the Romberg extrapolation, and has since been replaced by quadl as of Version 7, Release 14 [The Mathworks 2005].
}

ACM Computing Surveys, Vol. V, No. N, 20YY. 
by Garribba et al. [1978] in 1978 in their integrator SNIFF for Gauss-Legendre quadrature rules. They do not use Romberg extrapolation to refine the approximation of the integral, but the use the error estimate to guess the optimal width of the sub-intervals in each unconverged interval.

Finally, in a 2001 paper, Gander and Gautschi [2001] present two recursive adaptive quadrature routines. The first routine, adaptsim is quite similar to Lyness' SQUANK (see Section 2.1). It computes the approximations $\mathrm{S}^{(1)}[a, b]$ and $\mathrm{S}^{(2)}[a, b]$ and uses them to extrapolate $\mathrm{NC}_{5}^{(1)}[a, b]$ as in (10). The globally relative local error estimate, however, is then computed as

$$
\varepsilon_{k}=\left|\mathrm{NC}_{5}^{(1)}\left[a_{k}, b_{k}\right]-\mathrm{S}^{(2)}\left[a_{k}, b_{k}\right]\right| /|\hat{I}|
$$

where $\hat{I}$ is a rough approximation to the global integral computed over a set of random nodes.

\subsection{Finite-Difference Based Error Estimators}

In a 1967 paper, Gallaher [1967] presents a recursive adaptive quadrature routine based on the midpoint rule. In this algorithm, the interval is divided symmetrically into three sub-intervals with the width $h_{c}$ of the central sub-interval chosen randomly in $h_{c} \in\left[\frac{1}{6} h_{k}, \frac{1}{2} h_{k}\right], h_{k}=\left(b_{k}-a_{k}\right)$.

The integrand $f(x)$ is evaluated at the center of each sub-interval and used to compute the midpoint rule therein. Since the error of the midpoint rule is proportional to the second derivative of $f(x)$, the local integration error can be estimated by computing the second divided difference of $f(x)$ over the three values $f_{1}, f_{2}$ and $f_{3}$ in the center of the sub-intervals. Instead of the difference formula, Gallaher uses the more compact approximation

$$
\varepsilon=14.6\left|f_{1}-2 f_{2}+f_{3}\right| \frac{b_{k}-a_{k}-h_{c}}{2} .
$$

In which the constant 14.6 is determined empirically.

Similarly, Ninomiya [1980] presents a recursive adaptive quadrature routine based on closed Newton-Cotes rules. He uses rules with $2 n+1$ nodes (results are given for 5,7 and 9 points) and notes that these have an error of the form

$$
\mathrm{NC}_{2 n-1}[a, b]-\int_{a}^{b} f(x) \mathrm{d} x=K_{2 n+1}(b-a)^{2 n+1} f^{(2 n)}(\xi), \quad \xi \in[a, b] .
$$

Instead of using the same quadrature rule on two or more sub-intervals to approximate the error as in Kuncir's and Lyness' error estimates, he adds two nodes in the center of the leftmost and the rightmost intervals.

Using $5+2,7+2$ and $9+2$ point stencils, he computes the error estimators, e.g.

$$
\mathrm{D}_{9+2}[a, b] \approx \frac{37(b-a)^{11}}{3066102400} f^{(10)}(\xi), \quad \xi \in[a, b]
$$

which approximate the scaled $2 n+1$ st derivative in the analytical error of the Newton-Cotes rules. 


\subsection{Coefficient-Based Error Estimators}

In 1969, O'Hara and Smith [1969] publish a recursive adaptive quadrature routine based on Clenshaw-Curtis quadrature rules [Clenshaw and Curtis 1960]. Their algorithm uses a cascade of error estimates based on pairs of Newton-Cotes and Clenshaw-Curtis quadrature rules and the final error estimate is computed as

$$
\varepsilon_{k}=\frac{32}{\left(6^{2}-9\right)\left(6^{2}-1\right)}\left[\left|\sum_{i=1}^{7}{ }^{\prime \prime}(-1)^{i-1} f_{l, i}\right|+\left|\sum_{i=1}^{7}(-1)^{i-1} f_{r, i}\right|\right]
$$

where $\Sigma^{\prime \prime}$ denotes a sum in which first and last terms are halved and where the $f_{l, i}$ and $f_{r, i}$ are the values of the integrand evaluated at the nodes of two 7-point Clenshaw-Curtis quadrature rules over the left and right halves of the interval respectively. These sums are the approximated Chebyshev coefficients $\tilde{c}_{6}$ of the integrand over the left and right half of the interval.

The error estimate (14) is derived by O'Hara and Smith [1968] based on the error estimation used by Clenshaw and Curtis [1960]. They start by writing the error of a Clenshaw-Curtis quadrature rule over $n+1$ nodes as

$$
\begin{aligned}
\int_{a}^{b} f(x) \mathrm{d} x & -\mathrm{CC}_{n+1}^{(1)}[a, b]= \\
(b-a) & {\left[\frac{16 n}{\left(n^{2}-1\right)\left(n^{2}-9\right)} c_{n+2}+\frac{32 n}{\left(n^{2}-9\right)\left(n^{2}-25\right)} c_{n+4}+\ldots\right] }
\end{aligned}
$$

where the $c_{k}$ are the exact Chebyshev coefficients of

$$
f(x)=\sum_{k=0}^{\infty} c_{k} T_{k}(x)
$$

where $T_{k}(x)$ is the $k$ th Chebyshev polynomial of the first kind.

They note that for most regular functions, the first term in (15) is often larger than the sum of the following terms.

They find that if they define the higher-order $\left|c_{2 i}\right|, i>n+1$ in terms of $\left|c_{n+2}\right|$ using the recurrence relation $\left|c_{i+2}\right|=K_{n}\left|c_{i}\right|$, then they can define $K_{n}$ for different $n$ such that the first term of (15) dominates the series. For the 7-point ClenshawCurtis rule, this value is $K_{6}=0.12$. If the relation $\left|c_{i+2}\right| \leq K_{n}\left|c_{i}\right|$ holds, then the error is bounded by twice the first term of (15)

$$
\left|\int_{a}^{b} f(x) \mathrm{d} x-\mathrm{CC}_{n+1}^{(1)}[a, b]\right| \leq(b-a) \frac{32 n}{\left(n^{2}-1\right)\left(n^{2}-9\right)}\left|c_{n+2}\right| .
$$

However, we do not know $c_{n+2}$, yet since we assume that the magnitude of the coefficients decays, we can assume that $\left|c_{n+2}\right|<\left|c_{n}\right| \approx \frac{1}{2}\left|\tilde{c}_{n}\right|$ and use $\frac{1}{2}\left|\tilde{c}_{n}\right|$. Since $\left|c_{n}\right|$ might be "accidentally small", they suggest, in [O'Hara and Smith 1968], as an error estimate

$$
\varepsilon=(b-a) \frac{16 n}{\left(n^{2}-1\right)\left(n^{2}-9\right)} \max \left\{\left|\tilde{c}_{n}\right|, 2 K_{n}\left|\tilde{c}_{n-2}\right|, 2 K_{n}^{2}\left|\tilde{c}_{n-4}\right|\right\} .
$$

Oliver [1972] presents a similar doubly-adaptive Clenshaw-Curtis quadrature routine using an extension of the error estimate of O'Hara and Smith (see Section 2.3). 
Instead of assuming a constant $K_{n}$ such that $\left|c_{i+2}\right| \leq K_{n}\left|c_{i}\right|$ where the $c_{i}$ are the Chebyshev coefficients of the integrand, as do O'Hara and Smith, Oliver approximates the smallest rate of decrease of the coefficients as

$$
K=\max \left\{\left|\frac{\tilde{c}_{n}}{\tilde{c}_{n-2}}\right|,\left|\frac{\tilde{c}_{n-2}}{\tilde{c}_{n-4}}\right|,\left|\frac{\tilde{c}_{n-4}}{\tilde{c}_{n-6}}\right|\right\}
$$

where the $\tilde{c}_{i}$ are the Chebyshev coefficients approximated over the nodes of the quadrature rule.

He also pre-computes a number of convergence rates $K_{n}(\sigma)$, which are the rates of decay required such that, for $n$ coefficients, $\sigma$ times the first term of the error expansion in (15) dominates the sum of the remaining terms. If $K$ is less than any $K_{n}(\sigma)$ for $\sigma=2,4,8$ or 16 , then the error estimate

$$
\varepsilon=\sigma(b-a) \frac{16 n}{\left(n^{2}-1\right)\left(n^{2}-9\right)} \max \left\{K\left|\tilde{c}_{n}\right|, K^{2}\left|\tilde{c}_{n-2}\right|, K^{3}\left|\tilde{c}_{n-4}\right|\right\},
$$

which is consistent with (16) by O'Hara and Smith, is used.

If $\varepsilon$ exceeds the required local tolerance $\tau_{k}$, the computed rate of decrease $K$ is compared to a pre-computed limit $K_{n}^{*}$. This limit is defined by Oliver [1971] as the rate of decrease of the Chebyshev coefficients as of which it is preferable to subdivide the interval as opposed to doubling the order of the quadrature rule. Therefore, if $K>K_{n}^{*}$, the interval is subdivided, otherwise the order of the Clenshaw-Curtis quadrature rule is doubled.

Finally, Berntsen and Espelid [1991] present an error estimator based on sequences of null rules. Introduced by Lyness [1965], a null rule $\mathrm{N}_{n}^{(k)}$ of degree $k$ is defined as a set of weights $u_{i}^{(k)}$ over the $n$ nodes $x_{i}, i=1 \ldots n$ such that

$$
\sum_{i=1}^{n} u_{i}^{(k)} x_{i}^{j}= \begin{cases}0, & j \leq k \\ \neq 0 & j=k+1\end{cases}
$$

i.e. the rule evaluates all polynomials of degree $j \leq k$ to 0 and the $(k+1)^{\text {st }}$ monomial to some non-zero value.

Berntsen and Espelid compute a sequence of orthonormal ${ }^{9}$ null rules of decreasing degree $\mathrm{N}_{n}^{(n-1)}, \mathrm{N}_{n}^{(n-2)}, \ldots, \mathrm{N}_{n}^{(0)}$ which form an orthogonal basis $S_{n}$. Applying the null rules to the integrand $f(x)$ we obtain the interpolation coefficients $e_{k}=$ $\mathrm{N}_{n}^{(k)}[a, b]=\sum_{i=1}^{n} u_{i}^{(k)} f\left(x_{i}\right)$ of the integrand $f(x)$ onto $S_{n}$ such that

$$
f\left(x_{i}\right)=\frac{1}{\sum_{k=1}^{n} w_{k}^{2}} \sum_{k=0}^{n-1} e_{k} u_{i}^{(k)}, \quad i=1 \ldots n .
$$

To avoid "phase effects" as described in [Lyness and Kaganove 1976], the coefficients are then paired and the ratio of these pairs is computed

$$
r_{k}=\frac{E_{k}}{E_{k+1}}, \quad E_{k}=\left(e_{2 k}^{2}+e_{2 k+1}^{2}\right)^{1 / 2}, \quad k=0 \ldots n / 2-1 .
$$

\footnotetext{
${ }^{9}$ The null rules are normalized such that the norm of the coefficients is equal to the norm of the quadrature weights.
} 
The largest of the last $K$ ratios $r_{\max }=\max _{k} r_{k}$ is taken as an estimate of the convergence rate of the coefficients. If this ratio is larger than 1 then the function is assumed to be "non-asymptotic" in the interval and the largest $E_{k}$ is used as a local error estimate.

If $r_{\text {max }}$ is below 1 yet still above some critical value $r_{\text {critical }}$, the function is assumed to be "weakly asymptotic" and the value of the next-highest coefficient $E_{n / 2+1}$ and thus the local error - is estimated using

$$
\varepsilon_{k}=10 r_{\max } E_{n / 2-1}
$$

Finally, if $r_{\max }$ is below the critical ratio, then the function is assumed to be "strongly asymptotic" and the error is estimated using

$$
\varepsilon_{k}=10 r_{\text {critical }}^{1-\alpha} r_{\max }^{\alpha} E_{n / 2-1} .
$$

where $\alpha \geq 1$ is chosen to reflect, as Berntsen and Espelid state, "the degree of optimism we want to put into this algorithm."

Berntsen and Espelid implement and test this error estimate using 21-point Gauss, Lobatto, Gauss-Kronrod and Clenshaw-Curtis quadrature rules as well as 61-point Gauss and Gauss-Kronrod rules, and later in DQAINT [Espelid 1992], based on QUADPACK's QAG (see Section 2.4), using the Gauss, Gauss-Lobatto and GaussKronrod rules over 21 nodes. This approach is then extended to Newton-Cotes rules of different degrees and tested against a number of different quadrature routines [Espelid 2002; 2003; 2004a; 2004b; 2007].

More recently, Balles and Trefethen [2004] and Pachón et al. [2009] use a similar approach in the Chebfun system, in which arbitrary functions are represented as single or piecewise interpolants over Chebyshev nodes. These interpolations are considered to be sufficiently accurate in each interval when the absolute values of the highest-degree coefficients drop below a given tolerance. The integral of these interpolants can then be computed using Clenshaw-Curtis quadrature over the interpolation nodes, resulting in an adaptive quadrature scheme of sorts, although this is not the only purpose of the Chebfun system.

\subsection{Gauss-Kronrod Based Error Estimators}

In 1973 both Patterson [1973] and Piessens [1973] publish adaptive quadrature routines based on Gauss quadrature rules and their Kronrod extensions [Kronrod 1965].

Piessens' algorithm, which is the first to follow the scheme in Algorithm 2, uses an error estimate of the form

$$
\varepsilon_{k}=\left|\mathrm{G}_{n}\left[a_{k}, b_{k}\right]-\mathrm{K}_{2 n+1}\left[a_{k}, b_{k}\right]\right|
$$

where $\mathrm{G}_{n}[a, b]$ is the $n$-point Gauss quadrature rule of degree $2 n-1$ and $\mathrm{K}_{2 n+1}[a, b]$ is the $2 n+1$ point Gauss-Kronrod extension of degree $3 n+1$ which is in turn used as the approximation to the integral. This is also the error estimate currently used in Matlab's quadgk [Shampine 2008].

Patterson's integrator takes a different approach, starting with a 3-point Gauss quadrature rule and using the Kronrod scheme to successively extend it to 7, 15, $31,63,127$ and 255 nodes, resulting in quadrature rules of degree $5,11,23,47,95$, 


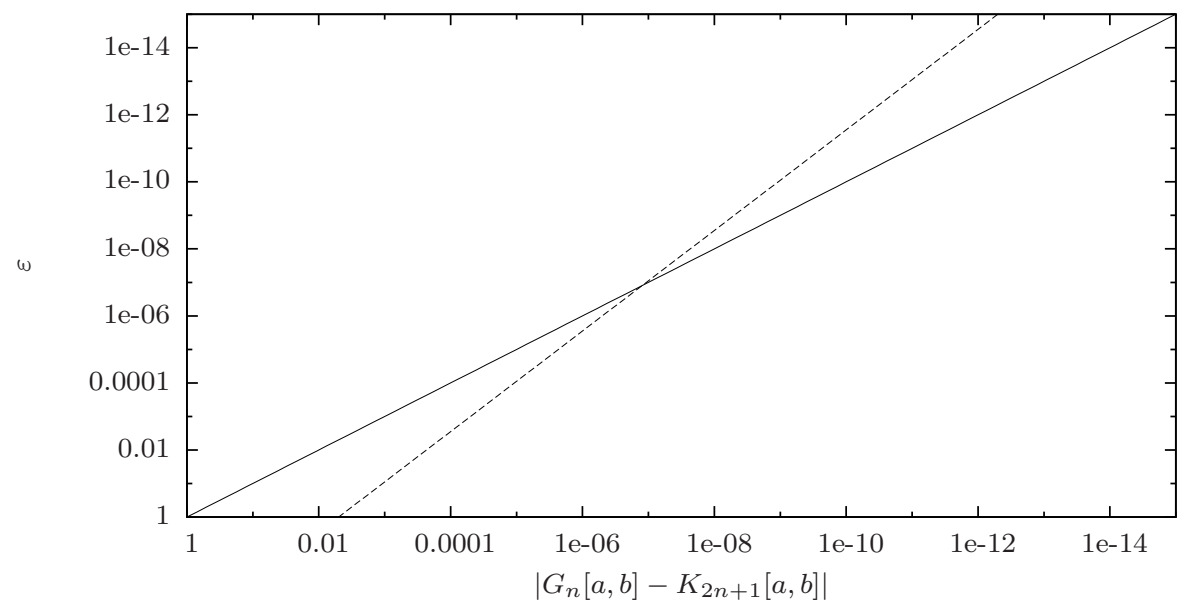

Fig. 1. The error measure $\left(200\left|\mathrm{G}_{n}[a, b]-\mathrm{K}_{2 n+1}[a, b]\right|\right)^{3 / 2}$ (dashed line) plotted as a function of $\left|\mathrm{G}_{n}[a, b]-\mathrm{K}_{2 n+1}[a, b]\right|$.

191 and 383 respectively, until the globally relative error estimate

$$
\varepsilon_{k}=\left|\mathrm{K}_{n}\left[a_{k}, b_{k}\right]-\mathrm{K}_{2 n+1}\left[a_{k}, b_{k}\right]\right| /|\hat{I}|
$$

where $\mathrm{K}_{n}[a, b]$ is the Kronrod extension over $n$ nodes and $\mathrm{K}_{2 n+1}[a, b]$ its extension over $2 n+1$ nodes, is below the required tolerance. $\hat{I}$ is an initial approximation of the global integral generated by applying successive Kronrod extensions to the whole interval before subdividing.

In 1983, the most widely-used "commercial strength" quadrature subroutine library QUADPACK is published by Piessens et al. [1983]. The general adaptive quadrature subroutine QAG is an extension of Piessens' integrator, yet with a slight modification to the local error estimate

$$
\varepsilon_{k}=\tilde{I}_{k} \min \left\{1,\left(200 \frac{\left|\mathrm{G}_{n}\left[a_{k}, b_{k}\right]-\mathrm{K}_{2 n+1}\left[a_{k}, b_{k}\right]\right|}{\tilde{I}_{k}}\right)^{3 / 2}\right\}
$$

where the default value of $n$ is 10 and the value

$$
\tilde{I}_{k}=\int_{a_{k}}^{b_{k}}\left|f(x)-\frac{\mathrm{K}_{2 n+1}\left[a_{k}, b_{k}\right]}{b_{k}-a_{k}}\right| \mathrm{d} x
$$

which is also evaluated using the $\mathrm{K}_{2 n+1}[a, b]$ rule, is used, as described by Krommer and Überhuber [1998], as "a measure for the smoothness of $f$ on $[a, b]$ ".

The error measure is best explained graphically, as is done in Piessens et al. (Fig. 1). The exponent $\frac{3}{2}$ is determined experimentally and scales the error exponentially, with a break-even point at $1.25 \times 10^{-6}$ which is approximately relative machine precision for IEEE 754 32-bit floating point arithmetic. The scaling makes the estimate increasingly pessimistic for error estimates larger than $1.25 \times 10^{-6}$ and increasingly optimistic for error estimates below that threshold. 
This measure is further divided by $\sqrt{\tilde{I}_{k}}$. Krommer and Überhuber explain this as follows:

"If this ratio is small, the difference between the two quadrature formulas is small compared to the variation of $f$ on $[a, b]$; i.e., the discretization of $f$ in the quadrature formulas $\mathrm{G}_{n}$ and $\mathrm{K}_{2 n+1}$ is fine with respect to its variation. In this case, $\mathrm{K}_{2 n+1}$ can indeed be expected to yield a better approximation for If than $\mathrm{G}_{n}$."

Unfortunately, no further analysis is given in either [Piessens et al. 1983] or [Krommer and Überhuber 1998].

This local error estimate is re-used by Favati et al. [1991a], yet using pairs of "recursive monotone stable" (RMS) nested quadrature rules introduced by Favati et al. [1991b], which allow for function evaluations to be re-used after bisection, within a doubly-adaptive scheme.

Hasegawa et al. [2007] extend this approach by choosing bisection over increasing the degree of the quadrature rule when the ratio of two successive error estimates is larger than an empirically determined constant (as is suggested by Venter and Laurie [2002], see Section 3.2).

In 1984 Berntsen and Espelid [1984] suggest that instead of using the difference between a Gauss quadrature rule over $n$ points and its Kronrod extension over $2 n+1$ points, one could directly use a Gauss quadrature rule over $2 n+1$ points for the estimate of the integral. To estimate the error of this rule of degree $4 n+1$, they suggest removing one of the points and creating a new interpolatory quadrature rule $\mathrm{Q}_{2 n}[a, b]$ of degree $2 n-1$ over the remaining $2 n$ points:

$$
\varepsilon_{k}=\left|\mathrm{G}_{2 n+1}\left[a_{k}, b_{k}\right]-\mathrm{Q}_{2 n}\left[a_{k}, b_{k}\right]\right| .
$$

Since the degree of the rule $\mathrm{Q}_{2 n}[a, b]$ is the same as that of the Gauss quadrature rule $\mathrm{G}_{n}[a, b]$ used by Piessens (see Section 2.4), the error estimate is 0 for functions of up to the same algebraic degree of precision, yet the final estimate is $n$ degrees higher: $4 n+1$ for $\mathrm{G}_{2 n+1}[a, b]$ vs. $3 n+1$ for $\mathrm{K}_{2 n+1}[a, b]$. A further advantage is the relative ease with which the weights of the rule $Q_{2 n}[a, b]$ can be computed, as opposed to the effort required for the nodes and weights of the Kronrod extension.

Finally, the second routine by Gander and Gautschi [2001] (see Section 2.1), adaptlob, uses a 4-point Gauss-Lobatto rule $\mathrm{GL}_{4}^{(1)}[a, b]$ and its 7-point Kronrod extension $\mathrm{K}_{7}^{(1)}[a, b]$. The globally relative local error is computed, analogously to (11), as

$$
\varepsilon_{k}=\left|\mathrm{GL}_{4}^{(1)}\left[a_{k}, b_{k}\right]-\mathrm{K}_{7}^{(1)}\left[a_{k}, b_{k}\right]\right| /|\hat{I}| .
$$

If the tolerance is met, the approximation $\mathrm{K}_{7}^{(1)}[a, b]$ is used for the integral.

\subsection{Summary}

Summarizing, we can group the different linear error estimators in the following categories:

(1) $\varepsilon \sim\left|Q_{n}^{\left(m_{1}\right)}[a, b]-Q_{n}^{\left(m_{2}\right)}[a, b]\right|:$ Error estimators based on the difference between two estimates of the same degree yet of different multiplicity [Kuncir 1962; 
McKeeman 1962; McKeeman and Tesler 1963; McKeeman 1963; Lyness 1969; 1970; Malcolm and Simpson 1975; Forsythe et al. 1977].

(2) $\varepsilon \sim\left|Q_{n_{1}}[a, b]-Q_{n_{2}}[a, b]\right|:$ Error estimators based on the difference between two estimates of different degree [Patterson 1973; Piessens 1973; Piessens et al. 1983; Hasegawa et al. 2007; Berntsen and Espelid 1984; Favati et al. 1991a; Gander and Gautschi 2001; O'Hara and Smith 1969].

(3) $\varepsilon \sim\left|f^{(n)}(\xi)\right|$ : Error estimators based on directly approximating the derivative in the analytic error term [Gallaher 1967; Garribba et al. 1978; Ninomiya 1980].

(4) $\varepsilon \sim\left|\tilde{c}_{n}\right|:$ Error estimators based on the estimate of the highest-degree coefficient of the function relative to some orthogonal base [O'Hara and Smith 1968; 1969; Oliver 1972; Berntsen and Espelid 1991; Espelid 1992; 2002; 2004a; 2004b; 2007].

Already in 1985, Laurie [1985] shows that the first three categories are, in essence, identical. Consider Kuncir's error estimate (see Section 2.1, (3)) from the first category (without the relative scaling), which can be viewed as a 5-point "rule" (or linear functional) over the nodes used by $\mathrm{S}^{(1)}[a, b]$ and $\mathrm{S}^{(2)}[a, b]$.

Since both approximations evaluate polynomials of up to degree 3 exactly, their difference will be, when applied to polynomials of up to degree 3, zero. When applied to a polynomial of degree 4 or higher, the estimates will, in all but pathological cases, differ. This is, up to a constant factor, exactly what the 4th divided difference over the same 5 nodes computes ${ }^{10}$.

The same can be said of error estimates from the second category, such as the one used by Piessens (see Section 2.4) where the Gauss quadrature rule $\mathrm{G}_{n}[a, b]$ integrates all polynomials of degree up to $2 n-1$ exactly and its Kronrod extension $\mathrm{K}_{2 n+1}[a, b]$ integrates all polynomials of degree up to $3 n+1$ exactly. Since the approximation computed by these rules differ only for polynomials of degree $2 n$ and higher, the combined "rule" over the $2 n+1$ points behaves just as the $2 n t h$ divided difference would.

In these cases, the divided differences are unique ${ }^{11}$ (i.e. the $n$th difference over $n+1$ points), as are the quadrature rules. They therefore differ only by a constant factor. As a consequence, the first and second categories, are both equivalent to the third category, in which the lowest degree derivative of the error expansion are approximated explicitly.

In the fourth and final category we again find finite differences, namely in Berntsen and Espelid's null rules (see Section 2.3), in which the coefficients $e_{k}$ relative to an orthogonal base are computed (see (20)). The highest-degree coefficient $e_{n-1}$, computed with the $(n-1)^{\text {st }}$ null rule over $n$ nodes is, as Berntsen and

\footnotetext{
${ }^{10}$ Note that this is also, up to a constant factor, the definition of a null-rule, as used by Berntsen and Espelid (see Section 2.3). Lyness [1965], who originally introduced the concept of null-rules, creates them explicitly from the difference of two quadrature rules, as is done in these error estimates implicitly.

${ }^{11}$ Not all error estimators in these categories, though, are identical up to a constant factor to the highest-degree divided differences over the same points. McKeeman's error estimator (see Section 2.1), for instance, approximates a 4th divided difference over 7 points, which is neither unique nor of the highest-possible degree. The same can be said of Forsythe, Malcolm and Moler's QUANC8 (see Section 2.1) and Patterson's successive Kronrod extensions (see Section 2.4).
} 
Espelid themselves note in [Berntsen and Espelid 1991], identical up to a constant factor to the $(n-1)^{\text {st }}$ divided difference over the same nodes. This value is combined with the $(n-2)^{\text {nd }}$ divided difference (see (21)), itself identical only up to a linear factor and used as an error estimate.

The same goes for the coefficients relative to any base computed over $n$ points, such as the coefficients $\tilde{c}_{i}$ of the Chebyshev polynomials used by O'Hara and Smith (see Section 2.3) and Oliver (see Section 2.3). The "rule" used to compute the highest-degree coefficients $((14))$ is identical up to a constant factor to the $n$th divided difference over the $n+1$ nodes used. While O'Hara and Smith use the highest-degree coefficient directly, Oliver uses $\mathrm{K}^{3}\left|\tilde{c}_{n-4}\right|$ (see (17) and (18)), which is related (i.e. no longer identical up to a constant factor) to the $(n-4)$ th divided difference.

We therefore establish that all linear error estimators presented in this section are equivalent in that they all use one or more divided difference approximations of the higher derivatives of the integrand. The quality of the error estimate therefore depends on the quality of these approximations.

In these estimates, problems may occur when the difference between two estimates or the magnitude of the computed coefficients is accidentally small i.e. the approximations used to compute the error estimate are too imprecise, resulting in a false small error estimate. This is often the case near singularities and discontinuities where the assumptions on which the error estimate is based, e.g. continuity and/or smoothness, do not hold.

\section{NON-LINEAR ERROR ESTIMATORS}

In the previous section, we considered error estimators that used only linear combinations of function values inside a single interval. In this section, we will consider methods that use function values or quadratures from one or more intervals or subintervals and which combine these values non-linearly to estimate the integration error.

\subsection{De Boor's CADRE Error Estimator}

In 1971, de Boor [1971] publishes the integration subroutine CADRE. The algorithm, which follows the scheme in Algorithm 1, generates a Romberg T-table [Bauer et al. 1963] with

$$
T_{\ell, i}=T_{\ell, i-1}+\frac{T_{\ell, i-1}-T_{\ell-1, i-1}}{4^{i}-1}
$$

in every interval. The entries in the T-table are used to decide whether to extend the table or bisect the interval ${ }^{12}$. After adding each $\ell$ th row to the table, a decision is made using the ratios

$$
R_{i}=\frac{T_{\ell-1, i}-T_{\ell-2, i}}{T_{\ell, i}-T_{\ell-1, i}}
$$

as to whether the integrand is linear, sufficiently smooth, discontinuous, singular or noisy inside the interval.

\footnotetext{
${ }^{12}$ Thus making it the first doubly-adaptive quadrature algorithm known to the author. 
If the integrand is assumed to be smooth $\left(R_{0}=4 \pm 0.15\right)$, the approximation $T_{\ell, i}$ is returned for the smallest $i \leq \ell$ such that the error

$$
\varepsilon_{k}=\left(b_{k}-a_{k}\right)\left|\frac{T_{\ell, i-1}-T_{\ell-1, i-1}}{4^{i}-1}\right| .
$$

is less than the required local tolerance. Otherwise, if a jump discontinuity is assumed $\left(R_{0}=2 \pm 0.01\right)$, the error is assumed to be bounded by the absolute difference of the two previous lowest-degree estimates:

$$
\varepsilon_{k}=\left|T_{\ell, 0}-T_{\ell-1,0}\right| \text {. }
$$

Finally, if the integrand is assumed to be singular $\left(R_{0} \in(1,4)\right.$ and is within $10 \%$ of the $R_{0}$ from the previous level $\left.\ell-1\right)$ and of the form $f(x)=(x-\xi)^{\alpha} g(x)$, where $\xi$ is near the edges of $\left[a_{k}, b_{k}\right]$ and $\alpha \in(-1,1)$. If this is the case, $R_{0}$ should be $\approx 2^{\alpha+i}$ and the T-Table is computed using "cautious extrapolation" by interleaving the normal updates in (29) with updates of the form

$$
T_{\ell, i}=T_{\ell, i-1}+\frac{T_{\ell, i-1}-T_{\ell-1, i-1}}{2^{\alpha+i}-1}
$$

where necessary. The error estimate is computed as in the smooth case (31) or as

$$
\varepsilon_{k}=\left(b_{k}-a_{k}\right)\left|\frac{T_{\ell, i-1}-T_{\ell-1, i-1}}{2^{\alpha+i}-1}\right|,
$$

depending on which column $i$ is considered.

The rationale for using the ratios $R_{i}$ (30) is based on the observation that the error of each entry of the T-table is, for sufficiently smooth integrands,

$$
\frac{1}{b-a} \int_{a}^{b} f(x) \mathrm{d} x-T_{\ell, i} \approx \kappa_{i}\left(2^{-(\ell-i)}\right)^{2 i+2} .
$$

The ratio $R_{i}$ can therefore be re-written as

$$
R_{i}=\frac{\kappa_{i}\left(2^{-(\ell-i-1)}\right)^{2 i+2}-\kappa_{i}\left(2^{-(\ell-2-i)}\right)^{2 i+2}}{\kappa_{i}\left(2^{-(\ell-i)}\right)^{2 i+2}-\kappa_{i}\left(2^{-(\ell-1-i)}\right)^{2 i+2}}=\frac{2^{2 i+2}-4^{2 i+2}}{1-2^{2 i+2}}=4^{i+1} .
$$

If this condition is actually satisfied (more or less), then de Boor considers it safe to assume that the difference between the two approximations $T_{\ell, i-1}$ and $T_{\ell, i}$ is a good bound for the error of $T_{\ell, i}$, as is computed in (31).

This error estimate for the regular case is itself, as defined at the beginning of this section, by no means non-linear. The reason for its inclusion in this category is the special treatment of integrable singularities in (33).

\subsection{Rowland and Varol's Modified Exit Procedure}

In 1972, Rowland and Varol [1972] publish an error estimator based on Simpson's compound rule. In their paper, they show that the "stopping inequality"

$$
\left|\mathrm{S}^{(m)}[a, b]-\mathrm{S}^{(2 m)}[a, b]\right| \geq\left|\mathrm{S}^{(2 m)}[a, b]-\int_{a}^{b} f(x) \mathrm{d} x\right|
$$


is valid if $f^{(4)}(x)$ is of constant sign for $x \in[a, b]$. They also show that under certain conditions there exists an integer $m_{0}$ such that the inequality is valid for all $m \geq m_{0}$.

They note that for the compound Simpson's rule

$$
\frac{\mathrm{S}^{(m)}[a, b]-\mathrm{S}^{(2 m)}[a, b]}{\mathrm{S}^{(2 m)}[a, b]-\mathrm{S}^{(4 m)}[a, b]} \approx 2^{2 q}
$$

holds, where usually $q=2$. This condition is used to test if $m$ is indeed large enough, much in the same way as de Boor's CADRE does (see (30)) to test for regularity. If this condition is more or less satisfied ${ }^{13}$ for any given $m$, then they suggest using

$$
\varepsilon_{k}=\frac{\left(\mathrm{S}^{(2 m)}\left[a_{k}, b_{k}\right]-\mathrm{S}^{(4 m)}\left[a_{k}, b_{k}\right]\right)^{2}}{\left|\mathrm{~S}^{(m)}\left[a_{k}, b_{k}\right]-\mathrm{S}^{(2 m)}\left[a_{k}, b_{k}\right]\right|} .
$$

This error estimate can be interpreted as follows: Let us assume that

$$
e_{m}=\left|\mathrm{S}^{(m)}[a, b]-\mathrm{S}^{(2 m)}[a, b]\right|
$$

is an estimate of the error of $\mathrm{S}^{(m)}[a, b]$. If we assume that the error estimates decrease at a constant rate $r$ when the multiplicity $m$ is doubled, then we can extrapolate the error of $\mathrm{S}^{(4 m)}[a, b]$ using

$$
e_{2 m}=r e_{m} \quad \Longrightarrow \quad r=\frac{e_{2 m}}{e_{m}}, \quad e_{4 m}=r e_{2 m} \quad \Longrightarrow \quad e_{4 m}=\frac{e_{2 m}^{2}}{e_{m}}
$$

which is exactly what is computed in (37).

A similar approach is taken by Venter and Laurie [2002], where instead of using compound Simpson's rules of increasing multiplicity, they use a sequence of stratified quadrature rules, described by Laurie [1992]. In their algorithm, the sequence of quadratures of increasing degree $\mathrm{Q}_{1}[a, b], \mathrm{Q}_{3}[a, b], \mathrm{Q}_{7}[a, b], \ldots, \mathrm{Q}_{2^{i}-1}[a, b]$ is computed and the differences of pairs of these rules are used to extrapolate the error of the highest-order $(i \mathrm{th})$ quadrature rule:

$$
\varepsilon_{k}=\frac{E_{i-1}^{2}}{E_{i-2}}, \quad E_{i}=\left|\mathrm{Q}_{2^{i}-1}[a, b]-\mathrm{Q}_{2^{i+1}-1}[a, b]\right| .
$$

\subsection{Laurie's Sharper Error Estimate}

In 1983, Laurie [1983] publishes a sharper error estimate based on two quadrature rules $\mathrm{Q}_{\alpha}[a, b]$ and $\mathrm{Q}_{\beta}[a, b]$ of degree $\alpha$ and $\beta$ respectively, where $\alpha>\beta$, or $\alpha=\beta$ and $\mathrm{Q}_{\alpha}[a, b]$ is assumed to be more precise than $\mathrm{Q}_{\beta}[a, b]$ :

$$
\varepsilon_{k}=\frac{\left(\mathrm{Q}_{\alpha}^{(2)}-\mathrm{Q}_{\beta}^{(2)}\right)\left(\mathrm{Q}_{\alpha}^{(2)}-\mathrm{Q}_{\alpha}^{(1)}\right)}{\mathrm{Q}_{\beta}^{(2)}-\mathrm{Q}_{\beta}^{(1)}-\mathrm{Q}_{\alpha}^{(2)}+\mathrm{Q}_{\alpha}^{(1)}}
$$

where the ranges $\left[a_{k}, b_{k}\right]$ are omitted for simplicity.

\footnotetext{
${ }^{13}$ Since their paper does not include an implementation, no specification is given to how close to a power of two this ratio has to be.

ACM Computing Surveys, Vol. V, No. N, 20YY.
} 


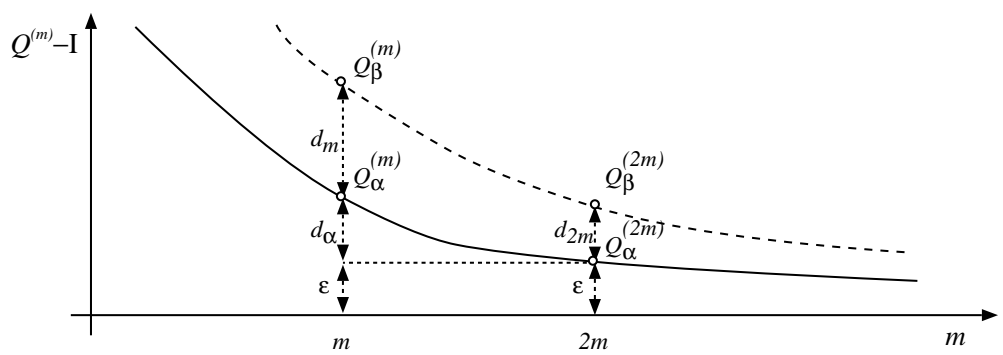

Fig. 2. The error of the quadrature rules $Q_{\alpha}^{(m)}$ (solid curve) and $Q_{\beta}^{(m)}$ (dotted curve) as a function of the number of panels or subdivisions $m$.

He shows that this error estimate is valid when

$$
\left|Q_{\alpha}^{(2)}-Q_{\alpha}^{(1)}\right|<\left|Q_{\beta}^{(2)}-Q_{\beta}^{(1)}\right| \text { and } 0 \leq \frac{Q_{\alpha}^{(2)}-I}{Q_{\alpha}^{(1)}-I} \leq \frac{Q_{\beta}^{(2)}-I}{Q_{\beta}^{(1)}-I}<1 .
$$

The former can be checked for in practice, yet the latter is impossible to verify since the exact integral $I$ must be known. These two conditions imply that the error of $\mathrm{Q}_{\alpha}[a, b]$ is smaller than and decreases at a faster rate than that of $\mathrm{Q}_{\beta}[a, b]$.

Laurie suggests a weaker condition that can be checked in practice, namely replacing $I$ by $\mathrm{Q}_{\alpha}^{(2)}\left[a_{k}, b_{k}\right]+\varepsilon_{k}$ in (41), resulting in

$$
0 \leq \frac{\mathrm{Q}_{\alpha}^{(2)}-\mathrm{Q}_{\beta}^{(2)}}{\mathrm{Q}_{\alpha}^{(1)}-\mathrm{Q}_{\beta}^{(1)}}<1 .
$$

Espelid and Sørevik [1989] show, however, that this weaker condition is often satisfied when (41) is not, which can lead to bad error estimates ${ }^{14}$.

The error estimate itself, based on these assumptions, is best explained graphically (see Fig. 2). The errors of both rules $Q_{\alpha}^{(m)}[a, b]$ and $Q_{\beta}^{(m)}[a, b]$ are assumed to decrease exponentially with the increasing number of panels or subdivisions $m$ :

$$
\mathrm{Q}_{\eta}^{(m)}-I=\kappa_{\eta}\left(\frac{b-a}{m}\right)^{\eta+2} f^{(\eta+1)}(\xi), \quad \xi \in[a, b] .
$$

We define the distances $\varepsilon, d_{2 m}, d_{\alpha}$ and $d_{m}$ using

$$
\begin{aligned}
& \mathrm{Q}_{\alpha}^{(2 m)}-I=\varepsilon, \quad \mathbf{Q}_{\beta}^{(2 m)}-I=\varepsilon+d_{2 m}, \\
& \mathbf{Q}_{\alpha}^{(m)}-I=\varepsilon+d_{\alpha}, \quad \mathbf{Q}_{\beta}^{(m)}-I=\varepsilon+d_{\alpha}+d_{m} .
\end{aligned}
$$

Inserting these terms into the second inequality in (41), we obtain

$$
\frac{\varepsilon}{\varepsilon+d_{\alpha}} \leq \frac{\varepsilon+d_{2 m}}{\varepsilon+d_{\alpha}+d_{m}} \Longrightarrow \varepsilon \leq \frac{d_{\alpha} d_{2 m}}{d_{2 m}-d_{m}}
$$

Resolving the distances using (43), we see that this bound is identical to the error estimate proposed by Laurie (40).

\footnotetext{
${ }^{14}$ Espelid and Sørevik show that this is the case when using the 10-point Gauss rule and its 21point Kronrod extension for $\mathrm{Q}_{\beta}^{(1)}$ and $\mathrm{Q}_{\alpha}^{(1)}$ respectively and integrating $\int_{1}^{2} 0.1 /\left(0.01+(x-\lambda)^{2}\right) \mathrm{d} x$ for $1 \leq \lambda \leq 2$.
} 
In 1991, Favati et al. [1991b] publish a similar error estimator, based on four quadratures $\mathrm{Q}_{\alpha}[a, b], \mathrm{Q}_{\beta}[a, b], \mathrm{Q}_{\gamma}[a, b]$ and $\mathrm{Q}_{\delta}[a, b]$ of degree $\alpha>\beta>\gamma>\delta$ that satisfy the relations

$$
\begin{aligned}
\left|I-\mathrm{Q}_{\alpha}\right| \leq\left|I-\mathrm{Q}_{\delta}\right|, \quad & \left|I-\mathrm{Q}_{\alpha}\right| \leq\left|I-\mathrm{Q}_{\gamma}\right| \\
& \left|I-\mathrm{Q}_{\alpha}\right| \leq\left|I-\mathrm{Q}_{\beta}\right|, \quad \frac{\left|I-\mathrm{Q}_{\alpha}\right|}{\left|I-\mathrm{Q}_{\gamma}\right|} \leq \frac{\left|I-\mathrm{Q}_{\beta}\right|}{\left|I-\mathrm{Q}_{\delta}\right|} .
\end{aligned}
$$

For any ordering of the four estimates $Q_{\alpha}, Q_{\beta}, Q_{\gamma}$ and $Q_{\delta}$ around the exact integral $I$, we can define the distances $d_{\alpha}=\left|Q_{\alpha}-I\right|, d_{\beta}, d_{\gamma}$ and $d_{\delta}$ depending on the configuration of the estimates around $I$, similarly to (43). The algorithm therefore first makes a decision as to which configuration is actually correct based on the differences between the actual estimates. Based on this decision, it computes the $d_{\alpha}, d_{\beta}, d_{\gamma}$ and $d_{\delta}$ or bounds them using the first three relations in (45) and inserts them into the final relation in (45) to extract an upper bound for $d_{\alpha}=\left|I-\mathrm{Q}_{\alpha}\right|$.

Favati et al. test this algorithm on a number of integrands and show that the milder conditions in (45), which do not require that successive estimates decrease monotonically, are satisfied more often than those of Laurie in (41).

\subsection{De Doncker's Adaptive Extrapolation Algorithm}

The probably best-known quadrature algorithm using non-linear extrapolation is published by de Doncker [1978]. The main idea of the algorithm is similar to that of the Romberg scheme: Given a basic quadrature rule $\mathrm{Q}_{n}[a, b]$, the series

$$
\mathrm{Q}_{n}^{(1)}[a, b], \mathrm{Q}_{n}^{(2)}[a, b], \mathrm{Q}_{n}^{(4)}[a, b], \ldots, \mathrm{Q}_{n}^{\left(2^{i}\right)}[a, b], \ldots
$$

converges exponentially, for large enough $i$ and sufficiently smooth $f(x)$, towards $\int_{a}^{b} f(x) \mathrm{d} x$.

In Romberg's scheme, $\mathbf{Q}_{n}^{(m)}[a, b]=\mathrm{T}^{(m)}[a, b]$, is the trapezoidal rule, and the limit of the series is extrapolated linearly using the the Romberg T-table. De Doncker's algorithm, however, differs in two main points: The 21-point Gauss-Kronrod rule is used as the basic rule $\mathrm{Q}_{n}^{(m)}[a, b]$ instead of the trapezoidal rule and the non-linear $\epsilon$-Algorithm [Wynn 1956] is used to extrapolate the limit of the series instead of the linear extrapolation in the Romberg T-table.

The algorithm, as described thus far, is not yet adaptive. The main (and new) trick is that instead of using $\mathbf{Q}_{n}^{(m)}[a, b]$, de Doncker uses approximations $\tilde{Q}_{n}^{(m)}[a, b]$. Each approximation $\tilde{Q}_{n}^{(m)}[a, b]$ is computed by iteratively picking out the subinterval of width greater than $h=(b-a) / m$ with the largest local error estimate

$$
\varepsilon_{k}=\left|\mathrm{G}_{10}\left[a_{k}, b_{k}\right]-\mathrm{K}_{21}\left[a_{k}, b_{k}\right]\right|
$$

which is the same local error estimate as first used by Piessens (see Section 2.4), and subdividing it until either the sum of the local error estimates $\varepsilon_{k}$ of all intervals of width larger than $h$ is smaller than the required tolerance or there are no more intervals of width larger than $h$ left to subdivide.

In her original paper, de Doncker does not give any details on how the $\epsilon$-Algorithm is applied or how the global error is estimated. In its implementation as QAGS in QUADPACK, the local error estimate (47) is replaced by the local error estimator 
used in the other QUADPACK-routines (see Section 2.4, (26)). A global error estimate is computed for the extrapolated $I_{i}$ using

$$
\varepsilon_{i}=\left|I_{i}-I_{i-1}\right|+\left|I_{i}-I_{i-2}\right|+\left|I_{i}-I_{i-3}\right|
$$

where $I_{i-1}, I_{i-2}$ and $I_{i-3}$ are the previous three estimates of the global integral.

\subsection{Summary}

Although most of the non-linear error estimators presented in this section differ significantly in their approach, they all rely on the same basic principle, namely the assumption that, for any quadrature rule $\mathrm{Q}^{(m)}[a, b]$, for sufficiently smooth $f(x)$ in the interval $x \in[a, b]$, the error can be written as

$$
\mathrm{Q}^{(m)}[a, b]-\int_{a}^{b} f(x) \mathrm{d} x \approx \kappa h^{\alpha}, \quad h=\frac{b-a}{m}
$$

where $\kappa$ depends on the basic quadrature rule $\mathrm{Q}$ and the higher derivatives of the integrand and $\alpha$ is the order of the error. In the most general case, (49) has three unknowns, namely the actual integral $I=\int_{a}^{b} f(x) \mathrm{d} x$, the scaling $\kappa$ and the order $\alpha$ of the error. The order $\alpha$ is usually assumed to be the order of the quadrature rule, but in the presence of singularities or discontinuities, this is not always the case. The three unknowns may be resolved using three successive approximations of increasing multiplicity:

$$
\begin{aligned}
\mathrm{Q}^{(m)} & =I+\kappa h^{\alpha} \\
\mathrm{Q}^{(2 m)} & =I+\kappa h^{\alpha} 2^{-\alpha} \\
\mathrm{Q}^{(4 m)} & =I+\kappa h^{\alpha} 4^{-\alpha}
\end{aligned}
$$

We can subtract (50) from (51) to isolate the error term

$$
\kappa h^{\alpha}=\frac{\mathrm{Q}^{(m)}-\mathrm{Q}^{(2 m)}}{1-2^{-\alpha}}=\frac{2^{\alpha}\left(\mathrm{Q}^{(m)}-\mathrm{Q}^{(2 m)}\right)}{2^{\alpha}-1} .
$$

Re-inserting this expression into (51), we obtain

$$
I=\mathrm{Q}^{(2 m)}-\frac{\mathrm{Q}^{(m)}-\mathrm{Q}^{(2 m)}}{2^{\alpha}-1}
$$

which is the linear extrapolation used in the Romberg T-table (for even integer values of $\alpha$ ) and also used by de Boor's CADRE (see Section 3.1, (32)), where the $\mathbf{Q}^{(m)}, \mathbf{Q}^{(2 m)}$ and $\mathbf{Q}^{(4 m)}$ are the T-table entries $T_{\ell-2, i}, T_{\ell-1, i}$ and $T_{\ell, i}$ respectively, for an unknown $\alpha$.

Inserting (53) into (51) and (52) and taking the difference of the two, we can extract

$$
2^{\alpha}=\frac{\mathrm{Q}^{(2 m)}-\mathrm{Q}^{(4 m)}}{\mathrm{Q}^{(m)}-\mathrm{Q}^{(2 m)}}
$$

which is the ratio $R_{i}$ used by de Boor $((30))$ to approximate the order of the error $\left(2^{\alpha+1}\right.$ therein).

Inserting both (53) and (54) into the last estimate, (52), we obtain

$$
I=\mathrm{Q}^{(4 m)}-\frac{\left(\mathrm{Q}^{(2 m)}-\mathrm{Q}^{(4 m)}\right)^{2}}{\mathrm{Q}^{(m)}-2 \mathrm{Q}^{(2 m)}+\mathrm{Q}^{(4 m)}}
$$


which is one step of the well-known Aitken $\Delta^{2}$-process [Aitken 1926].

The approach taken by Rowland and Varol (see Section 3.2) is almost identical, except that, instead of using the exact integral, they use

$$
\mathrm{Q}^{(m)}=\mathrm{Q}^{(2 m)}+\kappa h^{\alpha}, \quad \mathrm{Q}^{(2 m)}=\mathrm{Q}^{(4 m)}+\kappa h^{\alpha} 2^{-\alpha}, \quad \mathrm{Q}^{(4 m)}=I+\kappa h^{\alpha} 4^{-\alpha}
$$

to solve for $\kappa h^{\alpha}, 2^{-\alpha}$ and the exact integral $I$, resulting in their simpler error estimate (see (37)).

In a similar vein, Laurie (see Section 3.3) uses the four equations

$$
\begin{array}{ll}
\mathrm{Q}_{\alpha}^{(1)}=I+\kappa_{\alpha}(b-a)^{\alpha+2}, & \mathrm{Q}_{\alpha}^{(2)}=I+\kappa_{\alpha}(b-a)^{\alpha+2} 2^{-(\alpha+2)}, \\
\mathrm{Q}_{\beta}^{(1)}=I+\kappa_{\beta}(b-a)^{\beta+2}, & \mathrm{Q}_{\beta}^{(2)}=I+\kappa_{\beta}(b-a)^{\beta+2} 2^{-(\beta+2)}
\end{array}
$$

which are, however, under-determined, since there are 5 unknowns $\left(\kappa_{\alpha}, \kappa_{\beta}, \alpha, \beta\right.$ and $I$ ). To get a bound on the equation, Laurie therefore adds the conditions in (41), obtaining the inequality in (44) from which he constructs his error estimate.

Similarly, Favati, Lotti and Romani use the equations

$$
\begin{array}{ll}
\mathrm{Q}_{\alpha}=I+\kappa_{\alpha}(b-a)^{\alpha+2}, & \mathrm{Q}_{\beta}=I+\kappa_{\beta}(b-a)^{\beta+2}, \\
\mathrm{Q}_{\gamma}=I+\kappa_{\gamma}(b-a)^{\gamma+2}, & \mathrm{Q}_{\delta}=I+\kappa_{\delta}(b-a)^{\delta+2},
\end{array}
$$

which have 8 unknowns, and which can be solved together with the four conditions in (45).

Laurie and Venter's error estimator (see Section 3.2), differs in that, although similar in form to that of Rowland and Varol, the estimates

$$
\mathrm{Q}_{1}^{(1)}=I+\kappa_{1}(b-a)^{3}, \mathrm{Q}_{3}^{(1)}=I+\kappa_{3}(b-a)^{5}, \ldots, \mathrm{Q}_{255}^{(1)}=I+\kappa_{255}(b-a)^{257}
$$

form a set of $n$ equations in $n+1$ unknowns ( $I$ and the $n$ different $\kappa_{i}$, assuming, for simplicity, that the actual order of the error is that of the quadrature rule) which can not be solved as above.

In summary, these methods, i.e. Romberg's method, the Aitken $\Delta^{2}$-process and Rowland and Varol's extrapolation, take a sequence of initial estimates $\mathbf{Q}^{(m)}, \mathbf{Q}^{(2 m)}$, $\mathrm{Q}^{(4 m)}, \ldots$ and use them to create a sequence of improved estimates by removing the dominant error term as per (49). These approaches can, of course, be re-applied to the resulting sequence, thus eliminating the next dominant error term, and so on. This is exactly what is done in the columns of the Romberg T-table and in successive re-applications of the Aitken $\Delta^{2}$-process.

Instead of successively and iteratively removing the dominant term in the error, we could also simply model the error directly as the sum of several powers

$$
\mathrm{Q}^{(m)}-I \approx \kappa_{1} h^{\alpha_{1}}+\kappa_{2} h^{\alpha_{2}}+\cdots+\kappa_{N} h^{\alpha_{N}}, \quad h=\frac{b-a}{m}
$$

Since this equation has $2 N+1$ unknowns (the $N$ constants $\kappa_{i}$, the $N$ exponents $\alpha_{i}$ and the exact integral $I$ ), we need $2 N+1$ estimates to solve for them:

$$
\begin{aligned}
\mathrm{Q}^{(m)} & =I+\kappa_{1} h^{\alpha_{1}}+\kappa_{2} h^{\alpha_{2}}+\cdots+\kappa_{N} h^{\alpha_{N}} \\
\mathrm{Q}^{(2 m)} & =I+\kappa_{1} h^{\alpha_{1}} 2^{-\alpha_{1}}+\kappa_{2} h^{\alpha_{2}} 2^{-\alpha_{2}}+\cdots+\kappa_{N} h^{\alpha_{N}} 2^{-\alpha_{N}} \\
& \vdots \\
\mathrm{Q}^{\left(2^{2 N} m\right)} & =I+\kappa_{1} h^{\alpha_{1}} 2^{-2 n \alpha_{1}}+\kappa_{2} h^{\alpha_{2}} 2^{-2 n \alpha_{2}}+\cdots+\kappa_{N} h^{\alpha_{N}} 2^{-2 N \alpha_{N}}
\end{aligned}
$$


This non-linear system of equations does not appear to be an easy thing to solve, yet in [Kahaner 1972] Kahaner shows that, if we are only interested in $I$, this is exactly what the $\epsilon$-Algorithm [Wynn 1956] does. For an even number of approximations $2 N$, the algorithm computes the same approximation as in (58), yet only over the first $N-1$ terms, ignoring the first estimate $\mathrm{Q}^{(m)}$.

Keeping (58) in mind, de Doncker's error estimate (see Section 3.4, (48)) then reduces to

$$
\varepsilon_{i} \approx 2\left|\kappa_{N} h^{\alpha_{N}}\right|
$$

for $N=\lfloor i / 2\rfloor$, assuming that, ideally, for all estimates the right-most even column of the epsilon-table was used.

Generally speaking, we can say that all the error estimators presented herein assume that the error of a quadrature rule $\mathrm{Q}^{(m)}[a, b]$ behaves as in (58). The unknowns in this equation $\left(I=\int_{a}^{b} f(x) \mathrm{d} x, \kappa_{i}\right.$ and $\left.\alpha_{i}\right)$ can be solved for using several approximations $Q_{n}^{(m)}$.

In all these methods, the error estimate is taken to be the difference between the last estimate and the extrapolated value I of the integral. In the case of de Boor's CADRE, this is the difference between the last two entries in the bottom row of the modified T-table, and for Rowland and Varol (Section 3.2), Laurie (Section 3.3) and Favati, Lotti and Romani (Section 3.3), this is $\mathrm{Q}^{(4 m)}-I, \mathrm{Q}_{\alpha}^{(2)}-I$ and $\mathrm{Q}_{\alpha}-I$ respectively.

If the exponents $\alpha_{i}$ are known or assumed to be known, the resulting system is a linear system of equations. This is what Romberg's method does quite explicitly and what many of the error estimators in Section 2 do implicitly. If the exponents $\alpha_{i}$ are not known, the resulting system of equations is non-linear and can therefore only be solved for non-linearly.

The non-linear methods discussed here are therefore a conceptual extension of the linear error estimators presented earlier. As such, they are subject to the same problem of the difference between two estimates being accidentally small in cases where the assumptions in (49) or (58) do not actually hold, as is the case for singular or discontinuous integrands. The different error estimation techniques in this section differ only in the depth $N$ of the expansion and the use of additional constraints when the resulting system of equations is under-determined.

\section{A NEW ERROR ESTIMATOR}

In the following, we will present a new type of error estimator introduced by the author in [Gonnet 2010]. For the construction of this error estimator, we will begin with an explicit representation of the integrand. In almost all previously presented error estimators, the integrand itself is represented only by its approximated integral or, in the best of cases (see Section 2.3), only a few higher-order coefficients relative to some base.

By definition, every interpolatory quadrature rule implicitly constructs an interpolation polynomial $g_{n}(x)$ of degree $n-1$ of the integrand $f(x)$ at the nodes $x_{i}$, $i=1 \ldots n$ and computes the integral of the interpolation. This equivalence is easily demonstrated, as is done in many textbooks in numerical analysis ([Stiefel 1961; Rutishauser 1976; Gautschi 1997; Schwarz 1997; Ralston and Rabinowitz 1978] to 
name a few $)^{15}$.

For our new error estimate, we will represent the interpolant $g_{n}(x)$ explicitly as a weighted sum of orthonormal Legendre polynomials

$$
g_{n}(x)=\sum_{k=0}^{n-1} c_{k} p_{k}(x)
$$

The interpolant $g_{n}(x)$ interpolates the integrand $f(x)$ on the transformed interval from $[a, b]$ to $[-1,1]$ at the nodes $x_{i}, i=1 \ldots n$ :

$$
g_{n}\left(x_{i}\right)=\hat{f}\left(x_{i}\right)=f\left(\frac{a+b}{2}-\frac{a-b}{2} x_{i}\right), \quad x_{i} \in[-1,1] .
$$

Given the function values $\mathbf{f}=\left(\hat{f}\left(x_{1}\right), \hat{f}\left(x_{2}\right), \ldots, \hat{f}\left(x_{n}\right)\right)^{\top}$, we can compute the vector of coefficients $\mathbf{c}=\left(c_{0}, c_{1}, \ldots, c_{n-1}\right)^{\top}$ by solving the linear system of equations

$$
\mathbf{P c}=\mathbf{f}
$$

where the matrix $\mathbf{P}$ with $P_{i j}=p_{j}\left(x_{i}\right)$ on the left-hand side is a Vandermondelike matrix. The naive solution using Gaussian elimination is somewhat costly and may be unstable [Gautschi 1975]. However, several algorithms exist to solve this problem stably in $\mathcal{O}\left(n^{2}\right)$ operations for orthogonal polynomials satisfying a threeterm recurrence relation [Björck and Pereyra 1970; Higham 1988; 1990; Gonnet 2009b].

Given such a representation as in (60), the integral of $g_{n}(x)$ can be computed as

$$
\int_{-1}^{1} g_{n}(x) \mathrm{d} x=\sum_{k=0}^{n-1} c_{k} \int_{-1}^{1} p_{k}(x) \mathrm{d} x=\sum_{k=0}^{n-1} c_{k} \omega_{k}=\boldsymbol{\omega}^{\top} \mathbf{c} .
$$

Using orthonormal Legendre polynomials, the coefficients are simply $\boldsymbol{\omega}^{\top}=(1 / \sqrt{2}, 0, \ldots, 0)$. We can formulate the integral approximation as the scalar product of the vector of coefficients $\mathbf{c}$ with a vector of weights $\boldsymbol{\omega}$ :

$$
\mathrm{Q}_{n}[a, b]=\frac{(b-a)}{2} \boldsymbol{\omega}^{\top} \mathbf{c} .
$$

Another useful feature of such a representation is that it can be easily transformed to a sub-interval. Let $c_{i}, i=0 \ldots n-1$ be the coefficients of the interpolation $g_{n}(x)$ in the interval $[a, b]$. Given the matrix $\mathbf{T}^{(\ell)}$ with entries

$$
T_{i, j}^{(\ell)}=\int_{-1}^{1} p_{j}(x) p_{i}\left(\frac{x-1}{2}\right) \mathrm{d} x
$$

we can compute the coefficients $c_{i}^{(\ell)}, i=0 \ldots n-1$ of the interpolation $g_{n}^{(\ell)}(x)$ over the left half of the interval $[a,(a+b) / 2]$ using $c^{(\ell)}=\mathbf{T}^{(\ell)} \mathbf{c}$ where the resulting

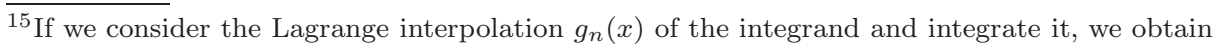

$$
\int_{a}^{b} g_{n}(x) \mathrm{d} x=\int_{a}^{b} \sum_{i=0}^{n} \ell_{i}(x) f\left(x_{i}\right) \mathrm{d} x=\sum_{i=0}^{n} f\left(x_{i}\right) \int_{a}^{b} \ell_{i}(x) \mathrm{d} x=\sum_{i=0}^{n} f\left(x_{i}\right) w_{i}
$$

where the $\ell_{i}(x)$ are the Lagrange polynomials and the $w_{i}$ are the weights of the resulting quadrature rule.

ACM Computing Surveys, Vol. V, No. N, 20YY. 
polynomial $g_{n}^{(\ell)}(x)$ over $[-1,1]$ is identical to $g_{n}(x)$ over $[-1,0]\left(g_{n}^{(\ell)}(x)=g_{n}\left(\frac{x-1}{2}\right)\right.$, $x \in[-1,1])$.

Analogously, we can create the matrix $\mathbf{T}^{(r)}$ such that $\mathbf{c}^{(r)}=\mathbf{T}^{(r)} \mathbf{c}$ are the coefficients of the right half of $g_{n}(x)$ transformed to $[-1,1]$. Such upper-triangular matrices can be constructed to transform $g_{n}(x)$ to any sub-interval.

A final useful feature is that given the coefficients $c_{i}, i=0 \ldots n-1$ of any interpolation $g_{n}(x)$, we can compute its $L_{2}$-norm using Parseval's theorem:

$$
\left[\int_{-1}^{1} g(x)^{2} \mathrm{~d} x\right]^{1 / 2}=\left[\sum_{i=0}^{n-1} c_{i}^{2}\right]^{1 / 2}=\|\mathbf{c}\|_{2}
$$

which is simply the Euclidean norm of the vector of coefficients c. In the following, we will use $\|\cdot\|$ to denote the 2-norm.

Instead of constructing our error estimate by approximating the difference of the integral of the interpolation $g_{n}(x)$ to the integral of the integrand $f(x)$ directly, as is done in practically all the methods presented in Section 2 and Section 3, we will consider the $L_{2}$-norm of the difference between the integrand and its interpolant:

$$
\varepsilon=\frac{b-a}{2}\left[\int_{-1}^{1}\left(\hat{f}(x)-g_{n}(x)\right)^{2} \mathrm{~d} x\right]^{1 / 2} .
$$

The proposed error estimate in (67) is, save for a constant factor of $\sqrt{2}$, an upper bound of the integration error of the interpolant $g_{n}(x)^{16}$

$$
\frac{b-a}{2}\left|\mathrm{Q}_{n}[-1,1]-\int_{-1}^{1} \hat{f}(x) \mathrm{d} x\right|=\frac{b-a}{2}\left|\int_{-1}^{1}\left(g_{n}(x)-\hat{f}(x)\right) \mathrm{d} x\right|
$$

and will only be zero if the interpolated integrand matches the integrand on the entire interval $\left(g_{n}(x)=\hat{f}(x), x \in[-1,1]\right)$. In such a case, the integral will also be computed exactly. The error (67) is therefore, assuming we can evaluate it reliably, not susceptible to "accidentally small" values.

Since we do not have an exact representation of the integrand $f(x)$, we can not compute (67) exactly. We can, however, generate a first trivial error estimate using two interpolations $g_{n_{1}}^{(1)}(x)$ and $g_{n_{2}}^{(2)}(x)$ of different degree where $n_{2}>n_{1}$. If we assume that $g_{n_{2}}^{(2)}(x)$ interpolates the integrand $f(x)$ much better than does $g_{n_{1}}^{(1)}(x)$, then we can assume that

$$
\hat{f}(x)-g_{n_{1}}^{(1)}(x) \approx g_{n_{2}}^{(2)}(x)-g_{n_{1}}^{(1)}(x)
$$

${ }^{16}$ This can be shown using the Cauchy-Schwarz inequality

$$
\left|\int_{-1}^{1} \phi(x) \psi(x) \mathrm{d} x\right|^{2} \leq \int_{-1}^{1}|\phi(x)|^{2} \mathrm{~d} x \int_{-1}^{1}|\psi(x)|^{2} \mathrm{~d} x .
$$

for $\psi(x)=1$ we obtain

$$
\left|\int_{-1}^{1} \phi(x) \mathrm{d} x\right|^{2} \leq 2 \int_{-1}^{1}|\phi(x)|^{2} \mathrm{~d} x
$$

and finally

$$
\left|\int_{-1}^{1} \phi(x) \mathrm{d} x\right| \leq \sqrt{2}\left(\int_{-1}^{1}|\phi(x)|^{2} \mathrm{~d} x\right)^{1 / 2}
$$


that is, that $f(x)$ on the left-hand side can be replaced with $g_{n_{2}}(x)$, similarly to Piessens' and Patterson's error estimates (see Section 2.4), in which the estimate from a higher-degree rule is used to estimate the error of a lower-degree rule. Taking the $L_{2}$-norm from the left-hand side of (68), we obtain

$$
\varepsilon_{1}=\frac{b-a}{2}\left\|\mathbf{c}^{(1)}-\mathbf{c}^{(2)}\right\|
$$

where $\mathbf{c}^{(1)}$ and $\mathbf{c}^{(2)}$ are the vectors containing the coefficients of the interpolants $g_{n_{1}}^{(1)}(x)$ and $g_{n_{2}}^{(2)}(x)$ respectively and $c_{i}^{(1)}=0$ where $i \geq n_{1}$.

This error estimate, however, is only valid for the lower-degree interpolation $g_{n_{1}}^{(1)}(x)$ and would over-estimate the error of the higher-degree interpolation $g_{n_{2}}^{(2)}(x)$ which we would use to compute the integral. For a more refined error estimate, we could consider the interpolation error

$$
\hat{f}(x)-g_{n}(x)=\frac{\hat{f}^{(n)}\left(\xi_{x}\right)}{n !} \pi_{n}(x), \quad \xi_{x} \in[-1,1]
$$

for any $n$ times continuously differentiable $f(x)$ where $\xi_{x}$ depends on $x$ and where $\pi_{n}(x)=\prod_{i=1}^{n}\left(x-x_{i}\right)$ is the Newton polynomial over the $n$ nodes of the quadrature rule:

Taking the $L_{2}$-norm on both sides of (70) we obtain

$$
\varepsilon=\left[\int_{-1}^{1}\left(g_{n}(x)-\hat{f}(x)\right)^{2} \mathrm{~d} x\right]^{1 / 2}=\left[\int_{-1}^{1}\left(\frac{\hat{f}^{(n)}\left(\xi_{x}\right)}{n !}\right)^{2} \pi_{n}^{2}(x) \mathrm{d} x\right]^{1 / 2} .
$$

Since $\pi_{n}^{2}(x)$ is, by definition, positive for any $x$, we can apply the mean value theorem of integration and extract the derivative resulting in

$$
\varepsilon=\left[\int_{-1}^{1}\left(g_{n}(x)-\hat{f}(x)\right)^{2} \mathrm{~d} x\right]^{1 / 2}=\left|\frac{\hat{f}^{(n)}(\xi)}{n !}\right|\left[\int_{-1}^{1} \pi_{n}^{2}(x) \mathrm{d} x\right]^{1 / 2}, \quad \xi \in[-1,1] .
$$

If we represent the polynomial $\pi_{n}(x)$ analogously to $g_{n}(x)$, as $\pi_{n}(x)=\sum_{k=0}^{n} b_{k} p_{k}(x)$, then we can compute its $L_{2}$-norm as $\|\mathbf{b}\|$, where $\mathbf{b}$ is the vector of the $n+1$ coefficients ${ }^{17} b_{k}$. Therefore, the terms on the right-hand side of (71), only the $n$th derivative of the integrand is unknown.

Given two interpolations of the integrand, $g_{n}^{(1)}(x)$ and $g_{n}^{(2)}(x)$, of the same degree yet not over the same set of nodes, if we assume that the derivative $f^{(n)}(\xi)$ is constant for $\xi \in[a, b]^{18}$, we can extract the unknown derivative as follows:

$$
g_{n}^{(1)}(x)-g_{n}^{(2)}(x)=\left|\frac{\hat{f}^{(n)}(\xi)}{n !}\right|\left(\pi_{n}^{(1)}(x)-\pi_{n}^{(2)}(x)\right)
$$

\footnotetext{
${ }^{17}$ Higham [1988] shows how the coefficients of a Newton-like polynomial can be computed relative to any orthogonal base.

${ }^{18}$ This assumption is a stronger form of the "sufficiently smooth" condition, which we will use only to construct the error estimator.

ACM Computing Surveys, Vol. V, No. N, 20 YY.
} 
where $\pi_{n}^{(1)}(x)$ and $\pi_{n}^{(2)}(x)$ are the $n$th Newton polynomials over the nodes of $g_{n}^{(1)}(x)$ and $g_{n}^{(2)}(x)$ respectively. Taking the $L_{2}$-norm on both sides of (72), we obtain

$$
\left|\frac{\hat{f}^{(n)}(\xi)}{n !}\right|=\frac{\left\|\mathbf{c}^{(1)}-\mathbf{c}^{(2)}\right\|}{\left\|\mathbf{b}^{(1)}-\mathbf{b}^{(2)}\right\|}
$$

from which we can construct an error estimate for either interpolation

$$
\left[\int_{-1}^{1}\left(g_{n}^{(k)}(x)-\hat{f}(x)\right)^{2} \mathrm{~d} x\right]^{1 / 2}=\frac{\left\|\mathbf{c}^{(1)}-\mathbf{c}^{(2)}\right\|}{\left\|\mathbf{b}^{(1)}-\mathbf{b}^{(2)}\right\|}\left\|\mathbf{b}^{(k)}\right\|, \quad k \in\{1,2\} .
$$

Note that for this estimate, we have made the assumption that the $n$th derivative is constant. We can't verify this directly, but we can verify if our computed $\left|\frac{f^{(n)}(\xi)}{n !}\right|$ (73) actually satisfies (70) for the nodes of the first interpolation by testing

$$
\left|g_{n}^{(2)}\left(x_{i}\right)-\hat{f}\left(x_{i}\right)\right| \leq \vartheta_{1}\left|\frac{f^{(n)}(\xi)}{n !}\right|\left|\pi_{n}^{(2)}\left(x_{i}\right)\right|, \quad i=1 \ldots n
$$

where the $x_{i}$ are the nodes of the interpolation $g_{n}^{(1)}(x)$ and the value $\vartheta_{1} \geq 1$ is an arbitrary relaxation parameter. If this condition is violated for any of the $x_{i}$, then we use the un-scaled estimate as in (69).

In practice, we can implement this error estimator in a recursive adaptive quadrature by first computing the $n$ coefficients $c_{k}$ of $g_{n}(x)$ in the interval $[a, b]$. The $n+1$ coefficients $b_{k}$ of the $n$th Newton polynomial over the nodes of the basic quadrature rule can be pre-computed.

For the first interval, no error estimate is computed. The interval is bisected and for the recursion on the left half of $[a, b]$, we compute ${ }^{19}$

$$
\mathbf{c}^{\text {old }}=\mathbf{T}^{(\ell)} \mathbf{c}, \quad \mathbf{b}^{\text {old }}=2^{n} \mathbf{T}^{(\ell)} \mathbf{b} .
$$

Inside the left sub-interval $[a,(a+b) / 2]$, we then evaluate the new coefficients $\mathbf{c}$. Given the old and new coefficients, we then compute the error estimate

$$
\varepsilon_{2}=\frac{(b-a)}{2} \frac{\left\|\mathbf{c}-\mathbf{c}^{\text {old }}\right\|}{\left\|\mathbf{b}-\mathbf{b}^{\text {old }}\right\|}\|\mathbf{b}\|
$$

\section{COMPARISON}

In the following, we will compare the performance of some of the error estimation techniques presented in $\S$ Section 2 and 3, including the new error estimator presented in Section 4.

\subsection{Methodology}

Whereas other authors [Casaletto et al. 1969; Hillstrom 1970; Kahaner 1971; Malcolm and Simpson 1975; Robinson 1979; Krommer and Überhuber 1998; Favati et al. 1991a] have focused on comparing different algorithms as a whole, using sets of functions chosen to best represent typical integrands, we will focus here only on

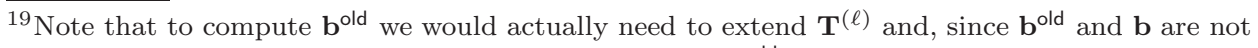
in the same interval, we have to scale the coefficients of $\mathbf{b}^{\text {old }}$ by $2^{n}$ so that Equation 70 holds for $g_{n}^{(2)}(x)$ in the sub-interval. 
specific error estimators and on integrands chosen such that they specifically should or should not cause the error estimator to fail.

For these test functions we will not consider the usual metrics of efficiency, i.e. number of function evaluations required for a given accuracy, but the number of correct estimates, false negatives and false positives for each error estimator when integrating functions which it should or should not integrate correctly, respectively.

We define a false positive as a returned error estimate which is below the required tolerance when the actual error is above the later. Likewise, a false negative is a returned error estimate which is above the required tolerance when the actual error is below the later.

In practical terms, false negatives are a sign that the error estimator is overly cautious and continues to refine an interval even though the required tolerance would already have been achieved. False positives, however, may cause the algorithm to fail completely: if the actual error in a sub-interval is larger than the global tolerance, no amount of excess precision in the other intervals will fix it and the result will be incorrect, save an identical false positive elsewhere of opposite sign.

The test integrands, along with an explanation of why they were chosen, are:

(1) $p_{n}(x)$ : The Chebyshev polynomial of degree $n$ in the interval $[-\alpha, \beta]$, where $\alpha$ and $\beta$ are chosen randomly in $(0,1]$ and $n$ is the degree of the quadrature rule for which the error estimate is computed ${ }^{20}$. The polynomial is shifted by +1 to avoid an integral of zero.

(2) $p_{n+1}(x)$ : Same as the function above, yet one degree above the degree of the quadrature rule. Although this integrand is, by design, beyond the degree of the quadrature rule, the error term (i.e. the $n+1^{\text {st }}$ derivative) is constant and can be extrapolated reliably ${ }^{21}$.

(3) $p_{n+2}(x)$ : Same as the function above, yet two degrees above the degree of the quadrature rule. By design, the $n+1$ st derivative is linear in $x$ and changes sign inside the interval, meaning that any attempt to extrapolate that derivative from two estimates of equal degree may fail.

(4) $d_{k}(x)$ : A function with a discontinuity at $x=\alpha$ in the $k$ th derivative, where $\alpha$ is chosen randomly in the interval of integration $[-1,1]$ for $k=0,1$ and 2 :

$$
\begin{aligned}
& d_{0}(x)= \begin{cases}0 & x<\alpha \\
1 & \text { otherwise }\end{cases} \\
& d_{1}(x)=\max \{0, x-\alpha\} \\
& d_{2}(x)=(\max \{0, x-\alpha\})^{2}
\end{aligned}
$$

Since all quadrature rules considered herein are interpolatory in nature and these integrands can not be reliably interpolated, these functions will only be

\footnotetext{
${ }^{20}$ For error estimates computed from the difference of two quadrature rules of different degree, the degree of the quadrature rule of lower degree is used since although the result rule of higher degree is effectively used for the returned integrand, the error estimate is usually understood to be that of the lower-degree rule.

${ }^{21}$ e.g. as is done implicitly in SQUANK (see Section 2.1, (9)) or explicitly in Ninomiya's error estimator (see Section 2.2, (13))

ACM Computing Surveys, Vol. V, No. N, 20YY.
} 
correctly integrated by chance ${ }^{22}$.

(5) $s(x)$ : A function with an integrable singularity at $x=\alpha$, where $\alpha$ is chosen randomly in $(-1,1)$ :

$$
s(x)=|x-\alpha|^{-1 / 2}
$$

As with the previous set of functions, this function can not be reliably interpolated and an interpolatory quadrature rule will produce a correct result only by chance ${ }^{23}$.

These functions were tested for 10000 realizations of the random parameters $\alpha$ and $\beta$ for each of the relative tolerances $\tau=10^{-1}, 10^{-3}, 10^{-6}, 10^{-9}$ and $10^{-12}$. Since most error estimators use absolute tolerances, the tolerance was set to the respective fraction of the integral. The following representative ${ }^{24}$ error estimators were implemented in Matlab (2007a, The MathWorks, Natick, MA.) ${ }^{25}$ and tested:

(1) Kuncir's error estimate (Section 2.1, (3)), where $n=3$ is the degree of the composite Simpson's rules used,

(2) Oliver's error estimate (Section 2.3, (18)), starting with a Clenshaw-Curtis rule of degree 3 , where $n=9$ is the degree of the second-last rule used and the first error estimate below tolerance is returned or $2 \tau$ if the interval is to be subdivided,

(3) QUADPACK's QAG error estimator (Section 2.4, (26)) using the 10-point Gauss quadrature rule with $n=19$ and its 21-point Kronrod extension,

(4) Berntsen and Espelid's null-rule error estimate (Section 2.3, (22) and (23)) using, as a basic quadrature rule, the 21-point Clenshaw-Curtis quadrature rule $^{26}$ with $n=21$ and values $K=3, r_{\text {critical }}=1 / 4$ and $\alpha=1 / 2$.

(5) Gander and Gautschi's error estimate as implemented in Matlab's quadl (Section 2.4, (28)) using the 4-point Gauss-Lobatto quadrature rule with $n=5$ and its 7-point Kronrod extension,

(6) Laurie's sharper error estimate (Section 3.3, (40)) using the 10-point Gauss quadrature rule with $n=19$ and its 21-point Kronrod extension for the two rules $Q_{\beta}$ and $Q_{\alpha}$ respectively, as suggested by Laurie [1985] himself,

(7) The trivial error estimate (Section 4, (69)) using the nodes of the $n=n_{1}=$ 11 and $n_{2}=21$-point Clenshaw-Curtis quadrature rules to compute the two interpolations $g_{n_{1}}^{(1)}(x)$ and $g_{n_{2}}^{(2)}(x)$ respectively.

(8) The more refined error estimate (Section 4, (76)) using the nodes of an 11point Clenshaw-Curtis quadrature rule with $n=10$ and one level of recursion to obtain $\mathbf{c}^{\text {old }}$, as well as 1.1 for the constant $\vartheta_{1}$ in (75).

\footnotetext{
${ }^{22}$ The only exception is CADRE (see Section 3.1), which attempts to detect jump discontinuities explicitly

${ }^{23}$ The only exception is again CADRE, which treats such singularities explicitly when detected (see Section 3.1, in cases where $\alpha$ is near the edges of the domain

${ }^{24}$ For compactness, the results for similar error estimators were omitted. The results for most other error estimators can be found in [Gonnet 2009a].

${ }^{25}$ The Matlab source code of each routine tested is available from this author online at http://people.inf.ethz.ch/gonnetp/csur/.

${ }^{26}$ the 21-point Gauss quadrature rule was also tried but left out since it produced worse results, i.e. more false positives.
} 


\begin{tabular}{lccccc} 
Function & $\tau=10^{-1}$ & $\tau=10^{-3}$ & $\tau=10^{-6}$ & $\tau=10^{-9}$ & $\tau=10^{-12}$ \\
\hline$p_{n}(x)$ & $100(0 / 0)$ & $100(0 / 0)$ & $100(0 / 0)$ & $100(0 / 0)$ & $100(0 / 0)$ \\
$p_{n+1}(x)$ & $65.67(0 / 34.33)$ & $8.49(0 / 21.14)$ & $0.38(0 / 0.76)$ & $0.01(0 / 0.02)$ & $0(0 / 0.01)$ \\
$p_{n+2}(x)$ & $51.07(0 / 48.93)$ & $8.44(0 / 15.77)$ & $0.50(0 / 1.07)$ & $0.03(0 / 0.11)$ & $0.02(0 / 0)$ \\
$d_{0}(x)$ & $16.58(0 / 22.27)$ & $0(0 / 0.35)$ & $0(0 / 0)$ & $0(0 / 0)$ & $0(0 / 0)$ \\
$d_{1}(x)$ & $44.92(0 / 29.98)$ & $0.73(0 / 1.59)$ & $0(0 / 0)$ & $0(0 / 0)$ & $0(0 / 0)$ \\
$d_{2}(x)$ & $54.30(0 / 22.66)$ & $5.74(0 / 7.16)$ & $0.22(0 / 0.12)$ & $0.01(0 / 0)$ & $0(0 / 0)$ \\
$s(x)$ & $0(33.05 / 17.11)$ & $0(0.42 / 0.20)$ & $0(0 / 0)$ & $0(0 / 0)$ & $0(0 / 0)$ \\
\hline
\end{tabular}

Table I. Results for Kuncir's 1962 error estimate.
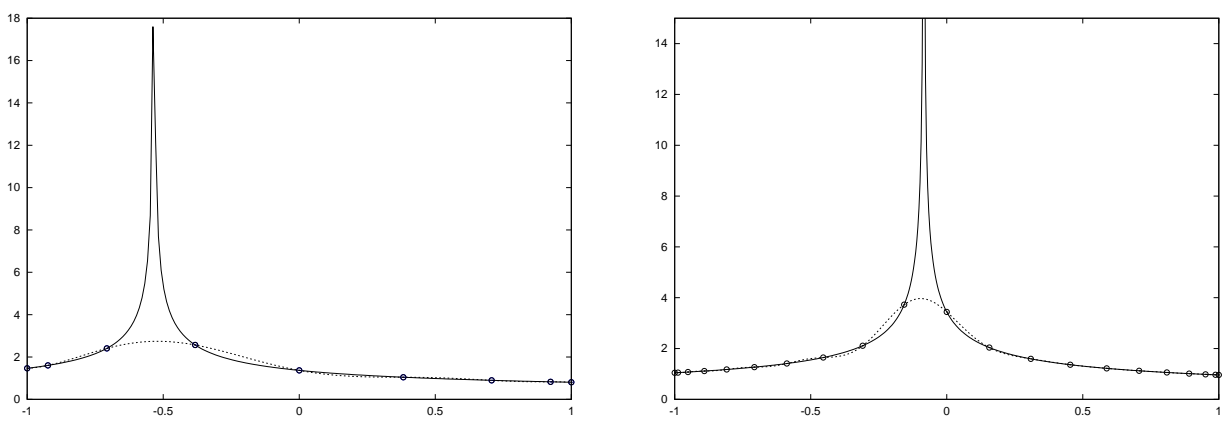

Fig. 3. The integrand assumed by the 9-point Clenshaw-Curtis rule (left, dotted) used in Oliver's 1972 error estimate and the 21-point Clenshaw-Curtis rule (right, dotted) used in Berntsen and Espelid's 1991 error estimate for the singular integrand $s(x)$ (solid).

\begin{tabular}{lccccc} 
Function & $\tau=10^{-1}$ & $\tau=10^{-3}$ & $\tau=10^{-6}$ & $\tau=10^{-9}$ & $\tau=10^{-12}$ \\
\hline$p_{n}(x)$ & $65.69(2.40 / 31.91)$ & $22.20(0.25 / 77.55)$ & $8.67(0 / 91.33)$ & $2.77(0 / 97.23)$ & $0.72(0 / 99.28)$ \\
$p_{n+1}(x)$ & $55.07(3.87 / 41.06)$ & $18.34(0.22 / 69.25)$ & $6.03(0 / 21.70)$ & $1.18(0 / 5.57)$ & $0.23(0 / 1.13)$ \\
$p_{n+2}(x)$ & $49.62(5.79 / 44.59)$ & $14.93(0.30 / 64.31)$ & $5.72(0 / 18.04)$ & $1.52(0 / 5.15)$ & $0.50(0 / 1.58)$ \\
$d_{0}(x)$ & $20.44(0 / 35.08)$ & $0(0 / 0.64)$ & $0(0 / 0)$ & $0(0 / 0)$ & $0(0 / 0)$ \\
$d_{1}(x)$ & $71.27(0.86 / 18.23)$ & $3.60(6.96 / 10.86)$ & $0(0 / 0.03)$ & $0(0 / 0)$ & $0(0 / 0)$ \\
$d_{2}(x)$ & $78.09(0 / 16.14)$ & $23.55(5.33 / 18.77)$ & $0.35(0 / 0.90)$ & $0.01(0 / 0.03)$ & $0(0 / 0)$ \\
$s(x)$ & $2.06(66.71 / 15.27)$ & $0(0.60 / 0.23)$ & $0(0 / 0)$ & $0(0 / 0)$ & $0(0 / 0)$ \\
\hline
\end{tabular}

Table II. Results for Oliver's 1972 error estimate.

\subsection{Results}

The results of the tests described in Section 5.1 are shown in Tables I to VIII. For each integrand and tolerance, the percentage of correct integrations is given (i.e. the error estimate and the actual error are both below the required tolerance), as well as, in brackets, the percentage of false positives and false negatives respectively.

Despite the low degree of the quadrature rule and its simplicity, Kuncir's error estimate (Section 2.1) performs rather well: almost all functions return no false positives and relatively few false negatives. Only the singularity returns false positives for $\tau=10^{-1}$ in more than a third of the cases.

Oliver's 1972 error estimate (Section 2.3) mis-predicts the errors for all three polynomials $p_{n}(x), p_{n+1}(x)$ and $p_{n+2}(x)$, due to the large higher-degree coefficients of the integrands. The false positives are cases where the doubly-adaptive algorithm exited after incorrectly predicting the error with a lower-order rule. This is also true for the discontinuities $d_{0}(x), d_{1}(x)$ and $d_{2}(x)$, which are detected well by the higherorder rules since the higher-degree Chebyshev coefficients become relatively large, yet fail when the error is mis-predicted by the lower-degree rules. The algorithm fails when integrating the singularity $s(x)$, since the coefficients of the interpolation 


\begin{tabular}{lccccc} 
Function & $\tau=10^{-1}$ & $\tau=10^{-3}$ & $\tau=10^{-6}$ & $\tau=10^{-9}$ & $\tau=10^{-12}$ \\
\hline$p_{n}(x)$ & $100(0 / 0)$ & $100(0 / 0)$ & $100(0 / 0)$ & $100(0 / 0)$ & $100(0 / 0)$ \\
$p_{n+1}(x)$ & $84.04(0 / 15.96)$ & $70.01(0 / 29.99)$ & $47.75(0 / 52.25)$ & $30.61(0 / 69.39)$ & $18.19(0 / 81.81)$ \\
$p_{n+2}(x)$ & $76.68(0 / 23.32)$ & $60.87(0 / 39.13)$ & $38.91(0 / 61.09)$ & $25.60(0 / 74.40)$ & $16.22(0 / 83.78)$ \\
$d_{0}(x)$ & $6.04(0.32 / 79.64)$ & $0.11(0.29 / 2.06)$ & $0(0.49 / 0)$ & $0(0.45 / 0)$ & $0(0.38 / 0)$ \\
$d_{1}(x)$ & $22.50(0.21 / 76.36)$ & $1.43(0.35 / 44.96)$ & $0.12(0.45 / 0.22)$ & $0.01(0.52 / 0)$ & $0(0.44 / 0)$ \\
$d_{2}(x)$ & $57.99(0.18 / 41.19)$ & $15.36(0.28 / 67.99)$ & $0.79(0.30 / 5.23)$ & $0.09(0.34 / 0)$ & $0.03(0.48 / 0)$ \\
$s(x)$ & $0.26(0.54 / 62.29)$ & $0(0.03 / 0.35)$ & $0(0 / 0)$ & $0(0 / 0)$ & $0(0 / 0)$ \\
\hline
\end{tabular}

Table III. Results for Piessens et al. 's 1983 error estimate.

\begin{tabular}{lccccc} 
Function & $\tau=10^{-1}$ & $\tau=10^{-3}$ & $\tau=10^{-6}$ & $\tau=10^{-9}$ & $\tau=10^{-12}$ \\
\hline$p_{n}(x)$ & $51.98(0 / 48.02)$ & $23.69(0 / 76.31)$ & $8.15(0 / 91.85)$ & $2.56(0 / 97.44)$ & $0.97(0 / 99.03)$ \\
$p_{n+1}(x)$ & $48.42(0 / 51.58)$ & $21.97(0 / 78.03)$ & $7.24(0 / 78.24)$ & $2.13(0 / 54.11)$ & $0.84(0 / 29.48)$ \\
$p_{n+2}(x)$ & $43.89(0 / 56.11)$ & $20.23(0 / 79.77)$ & $6.77(0 / 71.77)$ & $2.34(0 / 45.22)$ & $0.73(0 / 26.05)$ \\
$d_{0}(x)$ & $53.45(0 / 31.20)$ & $0(0 / 1.86)$ & $0(0 / 0)$ & $0(0 / 0)$ & $0(0 / 0)$ \\
$d_{1}(x)$ & $85.10(0 / 13.32)$ & $3.76(0 / 41.23)$ & $0(0 / 0.26)$ & $0(0 / 0)$ & $0(0 / 0)$ \\
$d_{2}(x)$ & $90.18(0 / 8.94)$ & $34.92(0 / 47.13)$ & $0.27(0 / 5.23)$ & $0(0 / 0.11)$ & $0(0 / 0)$ \\
$s(x)$ & $13.03(28.88 / 45.80)$ & $0(0 / 0.34)$ & $0(0 / 0)$ & $0(0 / 0)$ & $0(0 / 0)$ \\
\hline
\end{tabular}

Table IV. Results for Berntsen and Espelid's 1991 error estimate.

\begin{tabular}{lccccc} 
Function & $\tau=10^{-1}$ & $\tau=10^{-3}$ & $\tau=10^{-6}$ & $\tau=10^{-9}$ & $\tau=10^{-12}$ \\
\hline$p_{n}(x)$ & $100(0 / 0)$ & $100(0 / 0)$ & $100(0 / 0)$ & $100(0 / 0)$ & $100(0 / 0)$ \\
$p_{n+1}(x)$ & $80.08(0 / 19.92)$ & $17.69(0 / 82.31)$ & $0.56(0 / 99.44)$ & $0(0 / 100)$ & $0(0 / 99.99)$ \\
$p_{n+2}(x)$ & $68.15(0 / 31.85)$ & $17.88(0 / 82.12)$ & $2.46(0 / 97.54)$ & $0.33(0 / 99.67)$ & $0.08(0 / 99.92)$ \\
$d_{0}(x)$ & $10.33(0 / 39.32)$ & $0(0 / 0.59)$ & $0(0 / 0)$ & $0(0 / 0)$ & $0(0 / 0)$ \\
$d_{1}(x)$ & $63.43(2.32 / 23.63)$ & $0.70(1.33 / 9.97)$ & $0(0 / 0)$ & $0(0 / 0)$ & $0(0 / 0)$ \\
$d_{2}(x)$ & $68.98(0 / 19.77)$ & $8.69(0.03 / 25.79)$ & $0.31(0 / 0.13)$ & $0.02(0 / 0.01)$ & $0(0 / 0)$ \\
$s(x)$ & $0(44.15 / 22.67)$ & $0(0.50 / 0.22)$ & $0(0 / 0)$ & $0(0 / 0)$ & $0(0 / 0)$ \\
\hline
\end{tabular}

Table V. Results for Gander and Gautschi's 2001 error estimate.

often decay smoothly, misleading it to believe the integrand itself is smooth (see Fig. 3, left).

QUADPACK's error estimate (Section 2.4) does a very good job over all functions (Table III). The error estimate generates a high number of false negatives for the polynomials $p_{n+1}(x)$ and $p_{n+2}(x)$ since the quadrature rule used to approximate the integral is several degrees more exact than that for which the returned error estimate is computed. The few false positives are due to the error estimate's scaling of the error, causing it to under-predict the actual error and to cases where the discontinuity at $\alpha$ was outside of the open nodes of the quadrature rule. The false positives for the discontinuities $d_{0}(x), d_{1}(x)$ and $d_{2}(x)$ and the singularity $s(x)$ at $\tau=10^{-1}$ are due to accidentally small differences between the Gauss and GaussKronrod approximations.

Berntsen and Espelid's null-rule error estimate (Section 2.3) suffers from the same problems as Oliver's error estimate for the polynomial $p_{n}(x)$ : Although the integration is exact, the coefficients $\tilde{c}_{i}$ increase towards $i=n$, leading the algorithm to believe that the $n+1^{\text {st }}$ coefficient will be large when it is, in fact, zero. The algorithm mis-predicts the error for the singularity $s(x)$ for the same reason as Oliver's algorithm, namely that the coefficients of the polynomial interpolation decrease smoothly, falsely indicating convergence (see Fig. 3, right).

Gander and Gautschi's error estimate (Section 2.4) generates a high number of false negatives for $p_{n+1}(x)$ and $p_{n+2}(x)$, due to the higher degree of the estimate effectively returned. The error estimation returns some false positives for the discontinuities $d_{0}(x), d_{1}(x)$ and $d_{2}(x)$, as well as for the singularity $s(x)$, due to the difference between the two quadrature rules used being "accidentally small" 


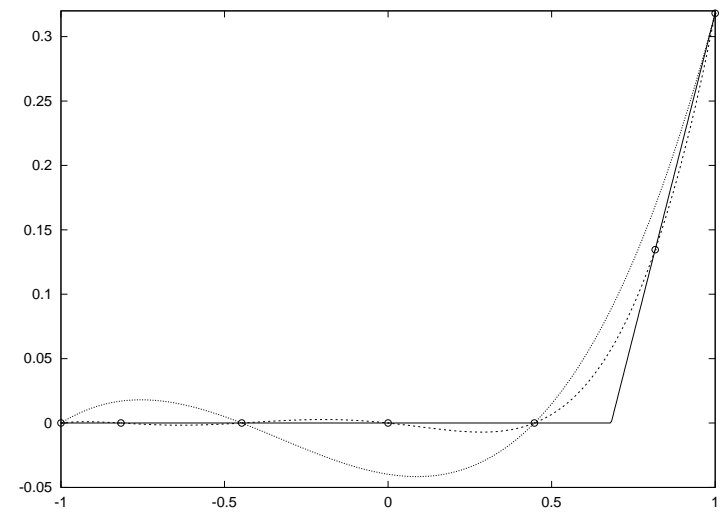

Fig. 4. The integrands assumed by the Gauss-Lobatto (dashed) and Gauss-Kronrod (dotted) quadrature rules in Gander and Gautschi's 2001 error estimate over the discontinuous integrand $d_{1}(x)$ (solid).

\begin{tabular}{lccccc} 
Function & $\tau=10^{-1}$ & $\tau=10^{-3}$ & $\tau=10^{-6}$ & $\tau=10^{-9}$ & $\tau=10^{-12}$ \\
\hline$p_{n}(x)$ & $100(0 / 0)$ & $100(0 / 0)$ & $100(0 / 0)$ & $100(0 / 0)$ & $100(0 / 0)$ \\
$p_{n+1}(x)$ & $100(0 / 0)$ & $100(0 / 0)$ & $100(0 / 0)$ & $100(0 / 0)$ & $100(0 / 0)$ \\
$p_{n+2}(x)$ & $100(0 / 0)$ & $100(0 / 0)$ & $100(0 / 0)$ & $100(0 / 0)$ & $100(0 / 0)$ \\
$d_{0}(x)$ & $30.26(0.09 / 62.46)$ & $0.12(0.09 / 3.93)$ & $0(0.18 / 0.01)$ & $0(0.20 / 0)$ & $0(0.24 / 0)$ \\
$d_{1}(x)$ & $36.78(0.07 / 62.75)$ & $24.67(3.78 / 48.51)$ & $0.25(1.14 / 0.55)$ & $0(0.41 / 0.01)$ & $0(0.46 / 0)$ \\
$d_{2}(x)$ & $44.81(0.11 / 54.70)$ & $40.21(0.94 / 51.18)$ & $3.52(4.74 / 15.25)$ & $0.14(0.13 / 0.16)$ & $0.03(0.32 / 0)$ \\
$s(x)$ & $25.01(0.06 / 64.82)$ & $0(4.52 / 0.52)$ & $0(0.03 / 0)$ & $0(0 / 0)$ & $0(0 / 0)$ \\
\hline
\end{tabular}

Table VI. Results for Laurie's 1983 error estimate.

\begin{tabular}{lccccc} 
Function & $\tau=10^{-1}$ & $\tau=10^{-3}$ & $\tau=10^{-6}$ & $\tau=10^{-9}$ & $\tau=10^{-12}$ \\
\hline$p_{n}(x)$ & $100(0 / 0)$ & $100(0 / 0)$ & $100(0 / 0)$ & $100(0 / 0)$ & $100(0 / 0)$ \\
$p_{n+1}(x)$ & $89.78(0 / 10.22)$ & $52.10(0 / 47.90)$ & $14.80(0 / 85.20)$ & $4.06(0 / 95.94)$ & $1.12(0 / 98.88)$ \\
$p_{n+2}(x)$ & $81.73(0 / 18.27)$ & $40.76(0 / 59.24)$ & $12.22(0 / 87.78)$ & $4.52(0 / 95.48)$ & $1.34(0 / 98.66)$ \\
$d_{0}(x)$ & $0(0 / 84.09)$ & $0(0 / 2.31)$ & $0(0 / 0)$ & $0(0 / 0)$ & $0(0 / 0)$ \\
$d_{1}(x)$ & $66.03(0 / 32.46)$ & $0.34(0 / 44.30)$ & $0(0 / 0.28)$ & $0(0 / 0)$ & $0(0 / 0)$ \\
$d_{2}(x)$ & $76.67(0 / 22.50)$ & $16.19(0 / 65.95)$ & $0.16(0 / 5.34)$ & $0.01(0 / 0.12)$ & $0(0 / 0)$ \\
$s(x)$ & $0(0 / 59.16)$ & $0(0 / 0.39)$ & $0(0 / 0)$ & $0(0 / 0)$ & $0(0 / 0)$ \\
\hline
\end{tabular}

Table VII. Results for Gonnet's 2009 trivial error estimate.

\section{(e.g. Fig. 4).}

Laurie's error estimate (Section 3.3) is exact even for the polynomials $p_{n+1}(x)$ and $p_{n+2}(x)$ : despite being of higher degree than the second-highest degree rule, the error of the highest-degree rule is correctly extrapolated. The discontinuities $d_{0}(x), d_{1}(x)$ and $d_{2}(x)$ and the singularity $s(x)$ are not always detected since the condition in (42) holds in some cases where the necessary condition in (41) does not, resulting in some false positives over all tolerances.

In both new error estimates described in Section 4, the errors of the polynomials $p_{n+1}(x)$ and $p_{n+2}(x)$ tend to be over-estimated as the computed $L_{2}$-norm is a somewhat pessimistic estimate of the integration error. What is notable is that these error estimates never under-estimated the error, resulting in no false positives at all. 


\begin{tabular}{lccccc} 
Function & $\tau=10^{-1}$ & $\tau=10^{-3}$ & $\tau=10^{-6}$ & $\tau=10^{-9}$ & $\tau=10^{-12}$ \\
\hline$p_{n}(x)$ & $100(0 / 0)$ & $100(0 / 0)$ & $100(0 / 0)$ & $100(0 / 0)$ & $100(0 / 0)$ \\
$p_{n+1}(x)$ & $100(0 / 0)$ & $100(0 / 0)$ & $58.76(0 / 41.24)$ & $17.49(0 / 82.51)$ & $5.15(0 / 94.85)$ \\
$p_{n+2}(x)$ & $83.30(0 / 16.70)$ & $58.78(0 / 41.22)$ & $28.18(0 / 71.08)$ & $9.05(0 / 46.17)$ & $3.03(0 / 14.26)$ \\
$d_{0}(x)$ & $0(0 / 81.48)$ & $0(0 / 2)$ & $0(0 / 0)$ & $0(0 / 0)$ & $0(0 / 0)$ \\
$d_{1}(x)$ & $68.87(0 / 27.89)$ & $0.40(0 / 54.34)$ & $0(0 / 0.10)$ & $0(0 / 0)$ & $0(0 / 0)$ \\
$d_{2}(x)$ & $82.21(0 / 15.81)$ & $17.88(0 / 58.11)$ & $0.22(0 / 5.08)$ & $0(0 / 0.07)$ & $0(0 / 0)$ \\
$s(x)$ & $0(0 / 59.19)$ & $0(0 / 0.33)$ & $0(0 / 0)$ & $0(0 / 0)$ & $0(0 / 0)$ \\
\hline
\end{tabular}

Table VIII. Results for Gonnet's 2009 refined error estimate.

\subsection{Summary}

According to the results using the chosen test integrands, the best two error estimators appear to be that of Piessens et al. (Section 2.4) which is the error estimator for the adaptive routines in the popular integration library QUADPACK, and the two new error estimators presented herein (Section 4).

The relatively few false positives returned by the QUADPACK error estimator may seem negligible in contrast with its efficiency (evidenced by the much smaller percentage of false negatives) compared to the new error estimate. We can verify this by evaluating the smooth integral

$$
\int_{1}^{2} \frac{0.1}{0.01+(x-\lambda)^{2}} d x
$$

first suggested by Lyness and Kaganove [1976], for which we compute 1000 realizations of the parameter $\lambda \in[1,2]$. We use both Piessens et al. 's error estimate and the two new error estimates as implemented for the previous tests in a recursive scheme as in Algorithm 1 with $\tau^{\prime}=\tau / \sqrt{2}$, to a relative precision of $\tau=10^{-9}$. On average, Piessens et al. 's error estimate requires 157 function evaluations while the new error estimates require 379 and 330 evaluations respectively - roughly more than twice as many. Both methods integrate all realizations to the required tolerance.

If we consider, however, the Waldvogel ${ }^{27}$ function

$$
W(x)=\int_{0}^{x}\left\lfloor e^{t}\right\rfloor \mathrm{d} t
$$

which we wish to evaluate to the relative precision $\tau=10^{-9}$ for 1000 realizations of $x \in[2.5,3.5]$ using both the error estimates of Piessens et al. and our new error estimators as described above, we get very different results. While Piessens et al. 's error estimator fails in roughly three quarters of all cases (753 failures out of 1000 , see Fig. 5), usually missing a sub-interval containing one or more discontinuities and using, on average, 29930 function evaluations, our new error estimators succeeds on every trial, using on average 31439 and 29529 function evaluations respectively. For this integrand, a single bad error estimate is sufficient for the entire computation to fail and, in this case, the cautious estimate pays off.

\section{CONCLUSIONS}

In this review we have analyzed a large part of error estimates for adaptive quadrature published in the last 45 years or so. We have shown that all these estimates

${ }^{27}$ This function was suggested to the author by Prof. Jörg Waldvogel. 


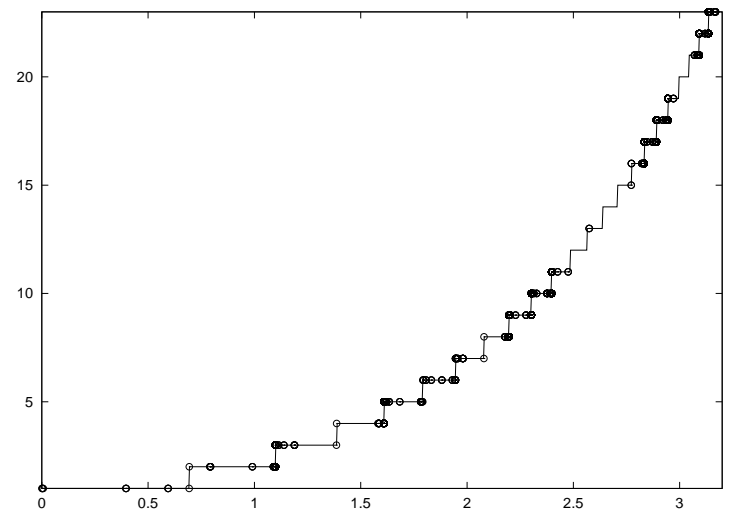

Fig. 5. Piessens et al. 's error estimate used to evaluate one realization of the Waldvogel-function. The circles mark the edges of the sub-intervals. Note that the integrand is not well resolved near $x \approx 1.4$ and $x \approx 2.6$.

can be reduced to either a linear or non-linear approximation of the integral and one or more error terms of the underlying quadrature rule:

$$
\mathrm{Q}_{n}^{(m)}[a, b]=\int_{a}^{b} f(x) \mathrm{d} x+\underbrace{\kappa_{1} h^{\alpha_{1}}+\kappa_{2} h^{\alpha_{2}}+\cdots+\kappa_{N} h^{\alpha_{N}}}_{=\varepsilon}, \quad h=\frac{b-a}{m} .
$$

For the linear error estimators discussed in Section 2, the exponents $\alpha_{i}, i=1 \ldots N$ are assumed to be known. For the non-linear error estimators discussed in Section 3, the $\alpha_{i}, i=1 \ldots N$ are not assumed to be known and are also approximated. In both cases, $N$ is usually 1 with the exception of de Boor's CADRE (see Section 3.1) and de Doncker's adaptive extrapolatory algorithm (see Section 3.4).

These error estimators all fail for the same reason, namely when the difference between two successive quadratures is "accidentally small". This can happen when the actual error contains more significant terms than the ones shown in (80).

The new error estimators presented in Section 4 are no different as they approximate the error for $N=1$ and a supposed $\alpha_{1}=n+1$. The main difference is that instead of using different approximations of the integral of different quadrature rules, we use the $L_{2}$-norm of the difference of the interpolating polynomials of different quadrature rules to approximate the unknown terms in (80). As we will see, this significantly reduces the probability of accidentally small differences, and thus avoid the major cause of failure of the other algorithms, as is demonstrated by the results in Section 5 .

The reason in this increased reliability is best explained by considering, for any error estimator, the set of integrands for which it will always fail. Consider the polynomials orthogonal with respect to the discrete product

$$
\left\langle p_{i}(x), p_{j}(x)\right\rangle=\sum_{k=1}^{n} p_{i}\left(x_{k}\right) p_{j}\left(x_{k}\right), \quad i, j=0 \ldots n
$$

where the $x_{k}$ are the nodes of the quadrature rule or the combined nodes of all the quadrature rules used in the computation of the error estimate in the interval. In 
the following, when we refer to a pair of functions being orthogonal, we understand them to be orthogonal with respect to the above product.

For any linear error estimate relying on the difference between two quadrature rules over the nodes $x_{i}$, the error estimate can be computed as

$$
\varepsilon=\sum_{i=1}^{n} \eta_{i} f\left(x_{i}\right)
$$

where the $\eta_{i}$ are the difference of the weights of the two quadrature rules used in the error estimate for each node ${ }^{28}$. Let $\eta(x)$ be the polynomial interpolating the $\eta_{i}$ at the nodes $x_{i}, i=1 \ldots n$. The error can then be computed as the product in (81) applied to the integrand $f(x)$ and the polynomial $\eta(x)$ :

$$
\varepsilon=\langle\eta(x), f(x)\rangle .
$$

Therefore, if the integrand $f(x)$ is of algebraic degree higher than that of the quadrature rule used - and will therefore not be correctly integrated - and the integrand $f(x)$ is orthogonal to the polynomial $\eta(x)$, then the linear error estimate will be zero and therefore it will fail.

For the error estimate of O'Hara and Smith (Section 2.3) and of Oliver (Section 2.3), which use more than one derivative, the error estimate fails when the integrand $f(x)$ is of higher algebraic degree than the basic quadrature rule and the coefficients $\tilde{c}_{n}, \tilde{c}_{n-2}$ and $\tilde{c}_{n-4}$ are zero (see (16)). This is the case when the integrand $f(x)$ is orthogonal to the Chebyshev polynomials $T_{n}(x), T_{n-2}(x)$ and $T_{n-4}(x)$.

For the error estimate of Berntsen and Espelid (Section 2.3), the error estimate fails when the integrand $f(x)$ is of higher algebraic degree than the basic quadrature rule and the integrand $f(x)$ is orthogonal to the last $2(K-1)$ null-rules ${ }^{29}$.

For the non-linear error estimates discussed in Section 3, the error estimates will fail under similar circumstances: In de Boor's CADRE (see Section 3.1), it is sufficient that the difference between two neighboring entries in the T-table is zero for the error estimate to fail. For a T-table of depth $\ell$, this engenders $\mathcal{O}\left(\ell^{2} / 2\right)$ different polynomials to which the integrand may be orthogonal to for the error estimate to fail.

In the case of Rowland and Varol's or Venter and Laurie's error estimates (see Section 3.2), a difference of zero between two consecutive pairs of rules is sufficient for the error estimate to fail and thus, as for the simple error estimators discussed above, for a sequence of $m$ rules, there are $m-1$ polynomials to which an integrand $f(x)$ may be orthogonal to for which the error estimator will always fail.

In Laurie's error estimate (see Section 3.3), either $Q_{\alpha}^{(2)}-Q_{\beta}^{(2)}$ or $Q_{\alpha}^{(2)}-Q_{\alpha}^{(1)}$ need to be zero for the estimate to fail, resulting in two polynomials to which the integrand may be orthogonal to for the error estimate to fail. Similarly, for Favati et al. 's error estimate (see Section 3.3), there are three such polynomials.

${ }^{28}$ The $\eta_{i}$ are, incidentally, the weights of a null rule, such as they are constructed by Lyness [1965]. ${ }^{29}$ In Berntsen and Espelid's original error estimate 2 null-rules are used to compute each $E_{k}$ from which the $K$ ratios $r_{k}$ (see (21)) are computed. It is, however, only necessary that the nominators of the ratios be zero, hence only $2(K-1)$ null-rules need to be zero for the estimate to be zero. 
Finally, for de Doncker's error estimate (see Section 3.4), the case is somewhat more complicated due to the global approach of the algorithm. Since it uses, locally, Piessens et al. 's local error estimate (see Section 2.4), it will fail whenever this estimate fails, making it vulnerable to the same family of integrands. Additionally, it will fail whenever the difference between two global estimates $\hat{Q}_{n}^{(m)}[a, b]-\hat{Q}^{(m-1)}[a, b]$ accidentally becomes zero, causing the algorithm to fail globally.

For both new error estimates presented here ((69) and (76)), the matter is a bit more complicated. Given two interpolations $g_{n_{1}-1}^{(1)}(x)$ and $g_{n_{2}-1}^{(2)}(x)$, with $n_{2} \geq n_{1}$, over the nodes $x_{i}^{(1)}, i=1 \ldots n_{1}-1$ and $x_{i}^{(2)}, i=1 \ldots n_{2}-1$ respectively, we define the joint set of $n_{u}$ nodes $x^{(u)}=x^{(1)} \cup x^{(2)}$ which we will use for the product in (81). Given the inverse Vandermonde-like matrices $\mathbf{U}^{(1)}=\left(\mathbf{P}^{(1)}\right)^{-1}$ and $\mathbf{U}^{(2)}=\left(\mathbf{P}^{(2)}\right)^{-1}$ of size $n_{1} \times n_{1}$ and $n_{2} \times n_{2}$ used to compute the coefficients of $g_{n_{1}}^{(1)}(x)$ and $g_{n_{2}}^{(2)}(x)$, we can stretch them to size $n_{2} \times n_{u}$ such that

$$
\mathbf{c}^{(1)}=\tilde{\mathbf{U}}^{(1)} \mathbf{f}^{(u)}, \quad \mathbf{c}^{(2)}=\tilde{\mathbf{U}}^{(2)} \mathbf{f}^{(u)}
$$

where $\tilde{\mathbf{U}}^{(1)}$ and $\tilde{\mathbf{U}}^{(2)}$ are the stretched matrices and $\mathbf{f}^{(u)}$ contains the integrand evaluated at the joint set of nodes $x^{(u)}$. For the error estimate $\left\|\mathbf{c}^{(1)}-\mathbf{c}^{(2)}\right\|$ to be zero, $\mathbf{f}^{(u)}$ must lie in the null-space of the $n_{2} \times n_{u}$ matrix

$$
\mathbf{U}^{(u)}=\left[\tilde{\mathbf{U}}^{(1)}-\tilde{\mathbf{U}}^{(2)}\right]
$$

which has rank $r_{u}$ equal to the smaller of the number of nodes not shared by both $x^{(1)}$ and $x^{(2)}$, i.e. $x^{(u)} \backslash\left\{x^{(1)} \cap x^{(2)}\right\}$ or $n_{2}$. For the error estimate to be zero, the product $\mathbf{U}^{(u)} \mathbf{f}^{(u)}$ must be zero. This is the case when the integrand $f(x)$ is of algebraic degree $>n_{2}$ and orthogonal to the $r_{u}$ polynomials generated by interpolating the values of the first $r_{u}$ rows of $\mathbf{U}^{(u)}$ at the nodes $x^{(u)}$. If, additionally, the integrand is of degree $>n_{2}$, then both error estimates will fail.

The space of functions that will cause any of the error estimators presented here to fail is, in essence, infinite, yet for each type of error estimator, this infinite space is subject to different restrictions. For the simple linear error estimators which compute a single divided difference, the space is restricted by a single orthogonality restriction. In the case of error estimators such as O'Hara and Smith's or Berntsen and Espelid's, the space is restricted by three or four ${ }^{30}$ orthogonality restrictions. Instead of being subject to one or more restrictions, the space of functions that will cause the non-linear error estimators discussed in Section 3 to fail is larger than that of the simple error estimators, since the integrand needs only to be orthogonal to any of a set of polynomials for the algorithm to fail. The set of functions for which they will fail is therefore the union of a set of functions, each subject to only one restriction. For our new error estimators, the number of restrictions depends on the number of nodes used. For the trivial error estimate $((69))$, if the nodes $x^{(1)} \subset x^{(2)}$ and $n_{2} \approx 2 n_{1}$ (i.e. if Clenshaw-Curtis or Gauss-Kronrod rule pairs are used), the number of restrictions will be $\approx n_{2} / 2$. For the more refined error estimate $((76))$, if the basic rule does not re-use more than $\lceil n / 2\rceil$ of its $n$ nodes in each sub-interval, the number of restrictions will be at least $n-1$.

\footnotetext{
${ }^{30}$ In Berntsen and Espelid's original error estimate, a constant $K=3$ is used. 
The new error estimates presented in Section 4 are therefore more reliable since the space of functions for which it will fail, albeit infinite, is more restricted than that of the other error estimators presented here. It is also interesting to note that if we were to increase the degree of the underlying quadrature rules in all our error estimates, the number of restrictions to the space of functions for which they will fail would not grow, whereas for our new error estimates, the number of restrictions grows linearly with the degree of the underlying quadrature rule.

Two adaptive quadrature algorithms implementing the new error estimates have been described and extensively tested in Gonnet [2010]. One of the algorithms presented therein has been implemented as cquad in both the GNU Scientific Library [Galassi et al. 2009] and as a part of GNU Octave [Eaton 2002].

\section{Acknowledgments}

The author would like to thank E.H.A. Venter, F.J. Smith, E. de Doncker, P. Davis, T.O. Espelid and R. Jaffe for their help in retrieving and understanding some of the older or less accessible publications included in this review as well as G.V. Milovanovic, B. Bojanov, G. Nikolov, A. Cvetkovic and G. Gonnet for the helpful discussions on quadrature, mathematics and everything else. Very special thanks go to W. Gander and J. Waldvogel, without who's immeasurable help this review wouldn't have gotten anywhere.

\section{REFERENCES}

Aitken, A. 1926. On Bernoulli's numerical solution of algebraic equations. Proceedings of the Royal Society of Edinburgh 46, 289-305.

Balles, Z. And Trefethen, L. N. 2004. An extensions of MATLAB to continuous functions and operators. SIAM Journal of Scientific Computing 25, 5, 1743-1770.

Bauer, F. L., Rutishauser, H., And Stiefel, E. 1963. New aspects in numerical quadrature. In Experimental Arithmetic, High Speed Computing and Mathematics, N. C. Metropolis, A. H. Taub, J. Todd, and C. B. Tompkins, Eds. American Mathematical Society, Providence, RI, 199-217.

Berntsen, J. And Espelid, T. O. 1984. On the use of Gauss quadrature in adaptive automatic integration schemes. BIT Numerical Mathematics 24, 239-242.

Berntsen, J. AND Espelid, T. O. 1991. Error estimation in automatic quadrature routines. ACM Transactions on Mathematical Software 17, 2, 233-252.

BJörck, Å. And Pereyra, V. 1970. Solution of Vandermonde systems of equations. Mathematics of Computation 24, 112, 893-903.

Casaletto, J., Pickett, M., And Rice, J. 1969. A comparison of some numerical integration programs. SIGNUM Newsletter 4, 3, 30-40.

Clenshaw, C. W. And Curtis, A. R. 1960. A method for numerical integration on an automatic computer. Numerische Mathematik 2, 197-205.

Davis, P. J. And Rabinowitz, P. 1967. Numerical Integration. Blaisdell Publishing Company, Waltham, Massachusetts.

DE BooR, C. 1971. CADRE: An algorithm for numerical quadrature. In Mathematical Software, J. R. Rice, Ed. Academic Press, New York and London, 201-209.

DE Doncker, E. 1978. An adaptive extrapolation algorithm for automatic integration. SIGNUM Newsletter 13, 2, 12-18.

EATon, J. W. 2002. GNU Octave Manual. Network Theory Limited, United Kingdom.

EsPelid, T. O. 1992. DQUAINT: An algorithm for adaptive quadrature over a collection of finite intervals. In Numerical Integration, T. O. Espelid and A. Genz, Eds. Kluwer Academic Publishers, Netherlands, 341-342. 
Espelid, T. O. 2002. Doubly adaptive quadrature routines based on Newton-Cotes rules. Tech. Rep. 229, Department of Informatics, University of Bergen, Norway. May.

Espelid, T. O. 2003. Doubly adaptive quadrature routines based on Newton-Cotes rules. BIT Numerical Mathematics 43, 319-337.

Espelid, T. O. 2004a. Extended doubly adaptive quadrature routines. Tech. Rep. 266, Department of Informatics, University of Bergen, Norway. February.

EsPelid, T. O. 2004b. A test of QUADPACK and four doubly adaptive quadrature routines. Tech. Rep. 281, Department of Informatics, University of Bergen, Norway. October.

Espelid, T. O. 2007. Algorithm 868: Globally doubly adaptive quadrature - reliable Matlab codes. ACM Transactions on Mathematical Software 33, 3, Article 21.

Espelid, T. O. And Sørevik, T. 1989. A discussion of a new error estimate for adaptive quadrature. BIT Numerical Mathematics 29, 293-294.

FAVATI, P., Lotti, G., And Romani, F. 1991a. Interpolatory integration formulas for optimal composition. ACM Transactions on Mathematical Software 17, 2, 207-217.

Favati, P., Lotti, G., And Romani, F. 1991b. Local error estimates in quadrature. BIT Numerical Mathematics 31, 102-111.

Forsythe, G. E., Malcolm, M. A., And Moler, C. B. 1977. Computer Methods for Mathematical Computations. Prentice-Hall, Inc., Englewood Cliffs, N.J. 07632.

Galassi, M., Davies, J., Theiler, J., Gough, B., Jungman, G., Alken, P., Booth, M., And Rossi, F. 2009. GNU Scientific Library Reference Manual, 3rd ed. Network Theory Ltd., United Kingdom.

Gallaher, L. J. 1967. Algorithm 303: An adaptive quadrature procedure with random panel sizes. Communications of the ACM 10, 6, 373-374.

Gander, W. 2006. Generating numerical algorithms using a computer algebra system. BIT Numerical Mathematics 46, 491-504.

Gander, W. And Gautschi, W. 2001. Adaptive quadrature - revisited. BIT 40, 1, 84-101.

Garribba, S., Quartapelle, L., and Reina, G. 1978. SNIFF: Efficient self-tuning algorithm for numerical integration. Computing 20, 363-375.

Gautschi, W. 1975. Norm estimates for inverses of Vandermonde matrices. Numerische Mathematik 23, 337-347.

Gautschi, W. 1997. Numerical Analysis, An Introduction. Birkhäuser Verlag, Boston, Basel and Stuttgart.

Gautschi, W. 2004. Orthogonal Polynomials: Computation and Approximation. Oxford University Press, Great Clarendon Street, Oxford OX2 6DP.

Gonnet, P. 2009a. Adaptive quadrature re-revisited. Ph.D. thesis, ETH Zürich, Switzerland.

Gonnet, P. 2010. Increasing the reliability of adaptive quadrature using explicit interpolants. ACM Transactions on Mathematical Software 37, 26:1-26:32.

Gonnet, P. G. 2009b. Efficient construction, update and downdate of polynomial interpolations based on polynomials satisfying a three-term recurrence relation. IMA Journal of Numerical Analysis Submitted.

Hasegawa, T., Hibino, S., Hosoda, Y., And Ninomiya, I. 2007. An extended doubly-adaptive quadrature method based on the combination of the Ninomiya and the FLR schemes. Numerical Algorithms 45, 1-4, 101-112.

Henrici, P. 1982. Essentials of Numerical Analysis, with Pocket Calculator Demonstrations. John Wiley \& Sons, Inc., New York.

Henriksson, S. 1961. Contribution no. 2: Simpson numerical integration with variable length of step. BIT Numerical Mathematics 1, 290.

Higham, N. J. 1988. Fast solution of Vandermonde-like systems involving orthogonal polynomials. IMA Journal of Numerical Analysis 8, 473-486.

Higham, N. J. 1990. Stability analysis of algorithms for solving confluent Vandermonde-like systems. SIAM Journal on Matrix Analysis and Applications 11, 1, 23-41.

ACM Computing Surveys, Vol. V, No. N, 20YY. 
Hillstrom, K. E. 1970. Comparison of several adaptive Newton-Cotes quadrature routines in evaluating definite integrals with peaked integrands. Communications of the ACM 13, 6, 362365.

Kahaner, D. K. 1971. 5.15 Comparison of numerical quadrature formulas. In Mathematical Software, J. R. Rice, Ed. Academic Press, New York and London, 229-259.

KahaneR, D. K. 1972. Numberical quadrature by the $\varepsilon$-algorithm. Mathematics of Computation 26, 119, 689-693.

Krommer, A. R. And Überhuber, C. W. 1998. Computational Integration. SIAM, Philadelphia.

Kronrod, A. S. 1965. Nodes and Weights of Quadrature Formulas - Authorized Translation from the Russian. Consultants Bureau, New York.

Kuncir, G. F. 1962. Algorithm 103: Simpson's rule integrator. Communications of the ACM 5, 6, 347.

LAURIE, D. P. 1983. Sharper error estimates in adaptive quadrature. BIT Numerical Mathematics 23, 258-261.

Laurie, D. P. 1985. Practical error estimation in numerical integration. Journal of Computational and Applied Mathematics 12-13, 425-431.

LAURIE, D. P. 1992. Stratified sequences of nested quadrature formulas. Quaestiones Mathematicae 15, 364-384.

LyNESS, J. N. 1965. Symmetric integration rules for hypercubes III: Construction of integration rules using null rules. Mathematics of Computation 19, 625-637.

LyNeSs, J. N. 1969. Notes on the adaptive Simpson quadrature routine. Journal of the ACM 16, 3, 483-495.

Lyness, J. N. 1970. Algorithm 379: SQUANK (Simpson Quadrature Used Adaptively - Noise Killed). Communications of the ACM 13, 4, 260-262.

Lyness, J. N. And Kaganove, J. J. 1976. Comments on the nature of automatic quadrature routines. ACM Transactions on Mathematical Software 2, 1, 170-177.

Lyness, J. N. And Kaganove, J. J. 1977. A technique for comparing automatic quadrature routines. The Computer Journal 20, 2, 170-177.

Malcolm, M. A. And Simpson, R. B. 1975. Local versus global strategies for adaptive quadrature. ACM Transactions on Mathematical Software 1, 2, 129-146.

McKeeman, W. M. 1962. Algorithm 145: Adaptive numerical integration by Simpson's rule. Communications of the ACM 5, 12, 604.

McKeeman, W. M. 1963. Algorithm 198: Adaptive integration and multiple integration. Communications of the ACM 6, 8, 443-444.

McKeeman, W. M. and Tesler, L. 1963. Algorithm 182: Nonrecursive adaptive integration. Communications of the ACM 6, 6, 315 .

Morrin, H. 1955. Integration subroutine - fixed point. Tech. Rep. 701 Note \#28, U.S. Naval Ordnance Test Station, China Lake, California. March.

NinomiYA, I. 1980. Improvements of adaptive Newton-Cotes quadrature methods. Journal of Information Processing 3, 3, 162-170.

O'Hara, H. And Smith, F. J. 1968. Error estimation in Clenshaw-Curtis quadrature formula. Computer Journal 11, 2, 213-219.

O'Hara, H. And Smith, F. J. 1969. The evaluation of definite integrals by interval subdivision. The Computer Journal 12, 2, 179-182.

Oliver, J. 1971. A practical strategy for the Clenshaw-Curtis quadrature method. Journal of the Institute of Mathematics and its Applications 8, 53-56.

Oliver, J. 1972. A doubly-adaptive Clenshaw-Curtis quadrature method. The Computer Journal 15, 2, 141-147.

Pachón, R., Platte, R., and Trefethen, L. N. 2009. Piecewise smooth chebfuns. IMA Journal of Numerical Analysis in Press.

Patterson, T. N. L. 1973. Algorithm 468: Algorithm for automatic numerical integration over a finite interval. Communications of the ACM 16, 11, 694-699.

Piessens, R. 1973. An algorithm for automatic integration. Angewandte Informatik 9, 399-401. 
Piessens, R., de Doncker-Kapenga, E., Überhuber, C. W., and Kahaner, D. K. 1983. QUADPACK A Subroutine Package for Automatic Integration. Springer-Verlag, Berlin.

Ralston, A. And Rabinowitz, P. 1978. A first course in Numerical Analysis. McGraw-Hill Inc., New York.

Robinson, I. 1979. A comparison of numerical integration programs. Journal of Computational and Applied Mathematics 5, 3, 207-223.

Rowland, J. H. AND VArol, Y. L. 1972. Exit criteria for Simpson's compound rule. Mathematics of Computation 26, 119, 699-703.

Rutishauser, H. 1976. Vorlesungen über numerische Mathematik, Band 1. Birkhäuser Verlag, Basel and Stuttgart.

Schwarz, H. R. 1997. Numerische Mathematik, Fourth ed. B. G. Teubner, Stuttgart. With a contribution by Jörg Waldvogel.

Shampine, L. 2008. Vectorized adaptive quadrature in matlab. Journal of Computational and Applied Mathematics 211, 2, 131-140.

Skeel, R. D. And Keiper, J. B. 1993. Elementary Numerical Computing with Mathematica. McGraw-Hill, Inc., New York.

Stiefel, E. 1961. Einführung in die numerische Mathematik. B. G. Teubner Verlagsgesellschaft, Stuttgart.

The Mathworks 2005. MATLAB 7.0 Release Notes. The Mathworks, Cochituate Place, 24 Prime Park Way, Natick, MA, USA.

Venter, A. And Laurie, D. P. 2002. A doubly adaptive integration algorithm using stratified rules. BIT Numerical Mathematics 42, 1, 183-193.

Villars, D. S. 1956. Use of the IBM 701 computer for quantum mechanical calculations II. overlap integral. Tech. Rep. 5257, U.S. Naval Ordnance Test Station, China Lake, California. August.

Wynn, P. 1956. On a device for computing the $e_{m}\left(s_{n}\right)$ transformation. Mathematical Tables and Other Aids to Computation 10, 54, 91-96. 


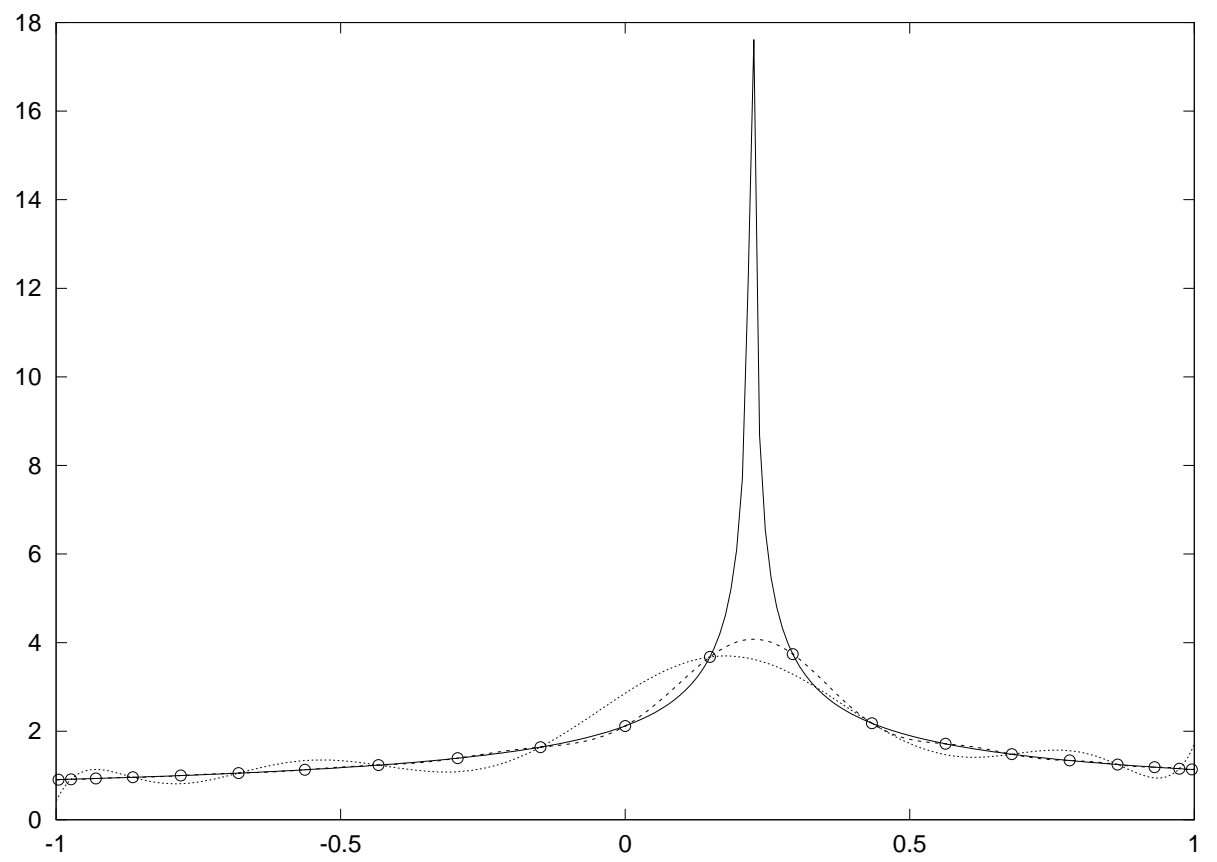

